\title{
xGASS: total cold gas scaling relations and molecular-to-atomic gas ratios of galaxies in the local Universe
}

\author{
Barbara Catinella, ${ }^{1 \star}$ Amélie Saintonge, ${ }^{2}$ Steven Janowiecki, ${ }^{1}$ Luca Cortese, ${ }^{1}$ \\ Romeel Davé, ${ }^{3}$ Jenna J. Lemonias, ${ }^{4}$ Andrew P. Cooper, ${ }^{5}$ David Schiminovich, ${ }^{4}$ \\ Cameron B. Hummels, ${ }^{6}$ Silvia Fabello, ${ }^{7}$ Katinka Geréb, ${ }^{8}$ Virginia Kilborn ${ }^{8}$ \\ and Jing Wang ${ }^{9}$ \\ ${ }^{1}$ International Centre for Radio Astronomy Research, M468, The University of Western Australia, Crawley, WA 6009, Australia \\ ${ }^{2}$ Department of Physics \& Astronomy, University College London, Gower Place, London WC1E 6BT, UK \\ ${ }^{3}$ Department of Physics \& Astronomy, University of the Western Cape, Bellville, Cape Town 7535, South Africa \\ ${ }^{4}$ Department of Astronomy, Columbia University, New York, NY 10027, USA \\ ${ }^{5}$ Department of Physics, Institute for Computational Cosmology, University of Durham, South Road, Durham DH1 3LE, UK \\ ${ }^{6}$ TAPIR, California Institute of Technology, Pasadena, CA 91125, USA \\ ${ }^{7}$ Autoliv Electronics Germany, Theodor-Heuss-Str 2, D-85221 Dachau, Germany \\ ${ }^{8}$ Centre for Astrophysics and Supercomputing, Swinburne University of Technology, Hawthorn, VIC 3122, Australia \\ ${ }^{9}$ Kavli Institute for Astronomy and Astrophysics, Peking University, Beijing 100871, China
}

Accepted 2018 January 4. Received 2017 December 18; in original form 2017 October 3

\begin{abstract}
We present the extended GALEX Arecibo SDSS Survey (xGASS), a gas fraction-limited census of the atomic hydrogen $\left(\mathrm{H}_{\mathrm{I}}\right)$ gas content of 1179 galaxies selected only by stellar mass $\left(M_{\star}=\right.$ $\left.10^{9}-10^{11.5} \mathrm{M}_{\odot}\right)$ and redshift $(0.01<z<0.05)$. This includes new Arecibo observations of 208 galaxies, for which we release catalogues and $\mathrm{H}_{\mathrm{I}}$ spectra. In addition to extending the GASS $\mathrm{H}$ i scaling relations by one decade in stellar mass, we quantify total (atomic+molecular) cold gas fractions and molecular-to-atomic gas mass ratios, $R_{\text {mol }}$, for the subset of 477 galaxies observed with the IRAM $30 \mathrm{~m}$ telescope. We find that atomic gas fractions keep increasing with decreasing stellar mass, with no sign of a plateau down to $\log M_{\star} / \mathrm{M}_{\odot}=9$. Total gas

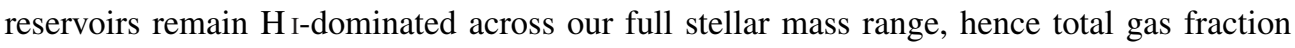
scaling relations closely resemble atomic ones, but with a scatter that strongly correlates with $R_{\mathrm{mol}}$, especially at fixed specific star formation rate. On average, $R_{\mathrm{mol}}$ weakly increases with stellar mass and stellar surface density $\mu_{\star}$, but individual values vary by almost two orders of magnitude at fixed $M_{\star}$ or $\mu_{\star}$. We show that, for galaxies on the star-forming sequence, variations of $R_{\mathrm{mol}}$ are mostly driven by changes of the $\mathrm{H}$ I reservoirs, with a clear dependence on $\mu_{\star}$. Establishing if galaxy mass or structure plays the most important role in regulating the cold gas content of galaxies requires an accurate separation of bulge and disc components for the study of gas scaling relations.
\end{abstract}

Key words: galaxies: evolution-galaxies: fundamental parameters-galaxies: ISM-radio lines: galaxies.

\section{INTRODUCTION}

The gas-star formation cycle is central to the formation and evolution of galaxies (see e.g. Leroy et al. 2008; Lilly et al. 2013 and review by Kennicutt \& Evans 2012). Understanding the complex interplay between the various components (such as multiphase neutral and ionized gas, and dust) of the interstellar medium (ISM; McKee \& Ostriker 1977; Cox 2005) and star formation as a function of galaxy properties, environment and cosmic time is a formidable task, which requires sensitive measurements across the electromagnetic spectrum and on multiple spatial scales for statistical data sets, as well as detailed numerical simulations to gain insights into the physical processes involved.

Even when we restrict ourselves to the global properties of galaxies in the local Universe, gathering the necessary data remains 
challenging. The main limitation comes from the paucity of measurements of the cold gas content ${ }^{1}$ for large, representative galaxy samples compared to optical, infrared, or ultraviolet surveys. Blind surveys of atomic hydrogen such as the H I Parkes All-Sky Survey (Barnes et al. 2001; Meyer et al. 2004; Wong et al. 2006), and the Arecibo Legacy Fast ALFA survey (ALFALFA; Giovanelli et al. 2005; Haynes et al. 2011) measured the H I content for $\sim 50000$ galaxies, but detect only the most gas-rich systems in most of their volume $\left(\sim 7000 \mathrm{deg}^{2}\right.$ and $z<0.06$ for ALFALFA). Samples of molecular hydrogen content, which is traced by carbon monoxide $\left({ }^{12} \mathrm{CO}\right.$, hereafter $\left.\mathrm{CO}\right)$ line emission, are almost two orders of magnitude smaller (Young et al. 1995; Saintonge et al. 2011; Boselli, Cortese \& Boquien 2014; Bothwell et al. 2014; Cicone et al. 2017). As a result, accurate constraints for key parameters such as the molecular-to-atomic gas mass ratio as a function of galaxy properties for unbiased samples are still scarce.

It is indeed generally accepted that atomic hydrogen has to transition into molecular phase in order to fuel star formation (Blitz \& Rosolowsky 2006; Bigiel et al. 2008; Leroy et al. 2008; Krumholz, McKee \& Tumlinson 2009), although molecular gas could just be tracing star formation, formed as the by-product of the gravitational collapse of atomic gas (Glover \& Clark 2012). The partition of total cold gas into $\mathrm{H}_{\mathrm{I}}$ and $\mathrm{H}_{2}$ and the efficiency of the atomic-tomolecular conversion are thus crucial quantities to measure in order to determine the physical processes regulating the star formation cycle in galaxies.

Substantial observing effort in the past decade went into measuring atomic and molecular gas masses for large samples of galaxies selected from optical surveys, and largely missed by H I-blind surveys (e.g. Young et al. 2011; Serra et al. 2012; Boselli et al. 2014).

Our GALEX Arecibo SDSS Survey (GASS; Catinella et al. 2010) was designed to investigate the main gas fraction scaling relations for a representative (in terms of $\mathrm{H}$ I content), stellar mass-selected sample of galaxies with stellar masses greater than $10^{10} \mathrm{M}_{\odot}$. The gas fraction limited nature of our observations means that integration times on each source were dictated by the request to reach gas fraction limits of $\sim 2$ percent, thus providing the most sensitive $\mathrm{H}_{\mathrm{I}}$ measurements for a large sample currently available. The combination of GASS on Arecibo and its follow-up program on the IRAM 30 m telescope (COLD GASS survey; Saintonge et al. 2011) resulted in a benchmark multiwavelength data set, including physical information about the stars and both atomic and molecular hydrogen gas phases in massive systems.

There were very good reasons to extend GASS and COLD GASS down to a stellar mass of $10^{9} \mathrm{M}_{\odot}$. First, these extensions would allow us to probe a crucial 'sweet spot' for understanding the physical processes that regulate the conversion of gas into stars and shape star-forming galaxies, without the additional complexities introduced by the presence of massive bulges and active galactic nuclei that are ubiquitous in the GASS stellar mass regime. Secondly, the scatter in the gas fraction scaling relations is expected to be driven by intrinsic properties of the discs, such as amount of angular momentum and stellar surface density (e.g. Fu et al. 2010). Again, testing

\footnotetext{
${ }^{1}$ By cold gas we refer to neutral hydrogen, both atomic and molecular. $\mathrm{H}_{\mathrm{I}}$ gas is typically found in two phases, a cold neutral medium $(T \lesssim 300 \mathrm{~K}$, best traced by $\mathrm{H}_{\mathrm{I}}$ in absorption) with a cloudy structure and a diffuse, warm neutral medium ( $T \gtrsim 5000 \mathrm{~K}$, providing the bulk of the $\mathrm{H}$ I seen in emission; Brinks 1990; Wolfire et al. 1995; Kalberla \& Kerp 2009); molecular hydrogen is found in dense clouds with lower temperatures $(T \sim 10-20 \mathrm{~K}$; Brinks 1990; Klessen \& Glover 2016).
}

this prediction with GASS is hampered by the presence of massive bulges, which could influence gas content as well (e.g. Martig et al. 2009). The new observations target a stellar mass regime that is dominated by star-forming discs, thus greatly alleviating these limitations. As showed by GASS, examining the scatter around the mean relations, and particularly its second-parameter dependencies, requires statistical samples of several hundred galaxies.

Here, we present the complete low-mass extension of GASS, hereafter GASS-low, which includes new Arecibo observations of 208 galaxies. The combination of GASS and GASS-low, which we refer to as the extended GASS (xGASS) survey, results in a representative sample of 1179 galaxies covering the $9<\log M_{\star} / \mathrm{M}_{\odot}<11.5$ stellar mass interval (see Section 2.2).

The companion extension of the molecular gas survey, $C O L D$ GASS-low, and the properties of the full xCOLD GASS sample are presented in Saintonge et al. (2017). Unlike the original GASS and COLD GASS surveys that were designed to explore the transition between star-forming and passive galaxies, these low-mass extensions aim to understand the basic physical processes governing star-forming galaxies.

This paper is organized as follows. In Section 2 we describe the sample selection and Arecibo observations of GASS-low galaxies, and combine these with GASS to obtain the xGASS representative sample. This includes the correct proportion of $\mathrm{H}$ I-rich ALFALFA galaxies that were not targeted to increase survey efficiency, and thus is representative of the $\mathrm{H}$ i properties of the galaxy population in our stellar mass and redshift intervals. We summarize in Section 3 the main properties of the XCOLD GASS survey, and discuss the overlap sample with both $\mathrm{H}_{\mathrm{I}}$ and $\mathrm{H}_{2}$ observations. Our $\mathrm{H} \mathrm{I}$, total gas, and $\mathrm{H}_{2} / \mathrm{H}$ I scaling relations are presented in Section 4; model comparisons are discussed in Section 5. We summarize our main findings and conclude in Section 6. The GASS-low data release can be found in Appendix A.

All the distance-dependent quantities in this work are computed assuming a cosmology with $H_{0}=70 \mathrm{~km} \mathrm{~s}^{-1} \mathrm{Mpc}^{-1}, \Omega_{\mathrm{m}}=0.3$ and $\Omega_{\lambda}=0.7$. We assume a Chabrier (2003) initial mass function. AB magnitudes are used throughout the paper.

\section{XGASS: THE EXTENDED GASS SURVEY}

\subsection{The low-mass extension of GASS}

\subsubsection{Sample selection and survey strategy}

The galaxies of the GASS low-mass extension were selected from a parent sample of 872 sources extracted from the intersection of the SDSS DR7 (Abazajian et al. 2009) spectroscopic survey, the GALEX Medium Imaging Survey (Martin et al. 2005) and projected ALFALFA footprints according to the following criteria:

(i) Stellar mass $9.0<\log M_{\star} / \mathrm{M}_{\odot}<10.2$

(ii) Redshift $0.01<z<0.02$.

Because GASS-low targets smaller galaxies than GASS, we lowered the redshift interval to ease their detection (GASS was limited to $0.025<z<0.05$ ). Galaxies in these stellar mass and redshift intervals have angular diameters smaller than 1 arcmin (as in GASS). Thus, our targets fit comfortably within a single SDSS frame and GALEX pointing, so that accurate photometry (and hence stellar masses and star formation rates) can be measured, and a single pointing of the IRAM $30 \mathrm{~m}$ telescope recovers an accurate total CO flux in most cases. 
For GASS, we imposed a flat stellar mass distribution for our targets, in order to ensure enough statistics at the high-mass end. Similarly, we sampled the stellar mass interval of GASS-low galaxies roughly evenly (see Section 2.2.1).

In order to optimize survey efficiency, we prioritized the observations of the galaxies lying within the ALFALFA 40 per cent (hereafter AA40; Haynes et al. 2011) footprint and/or galaxies already observed with the IRAM telescope. Galaxies with good quality $\mathrm{H}_{\mathrm{I}}$ detections already available from AA40 or the Cornell $\mathrm{H}_{\text {I }}$ digital archive (Springob et al. 2005, hereafter S05) were not reobserved (see Section 2.2 below).

Following the GASS strategy, we observed the targets until detected, or until a limit of a few per cent in gas mass fraction $\left(M_{\mathrm{HI}} / M_{\star}\right)$ was reached. Practically, we set a limit of $M_{\mathrm{HI}} / M_{\star}>0.02$ for galaxies with $\log \left(M_{\star} / \mathrm{M}_{\odot}\right)>9.7$, and a constant gas mass limit $\log \left(M_{\mathrm{HI}} / \mathrm{M}_{\odot}\right)=8$ for galaxies with smaller stellar masses. This corresponds to a gas fraction limit of $0.02-0.1$ for the whole sample.

Given the Hi mass limit assigned to each galaxy (set by its gas fraction limit and stellar mass), we computed the on-source observing time, $T_{\max }$, required to reach that value with our observing mode and instrumental setup, assuming a velocity width of $200 \mathrm{~km}$ $\mathrm{s}^{-1}$, smoothing to half width, and signal to noise of 5 . The $T_{\max }$ values thus obtained vary between 1 and $95 \mathrm{~min}$.

\subsubsection{Arecibo observations and data reduction}

GASS-low observations started in August 2012 and ended in May 2015. These were scheduled in 100 observing runs under Arecibo programs A2703 and A2749; the total telescope time allocation was $263 \mathrm{~h}$.

The observing mode and data reduction were the same as GASS. All the observations were carried out remotely in standard positionswitching mode, using the $L$-band wide receiver and the interim correlator as a backend. Two correlator boards with $12.5 \mathrm{MHz}$ bandwidth, one polarization, and 2048 channels per spectrum (yielding a velocity resolution of $1.4 \mathrm{~km} \mathrm{~s}^{-1}$ at $1370 \mathrm{MHz}$ before smoothing) were centred at or near the frequency corresponding to the SDSS redshift of the target. We recorded the spectra every second with 9-level sampling.

The data reduction, performed in the IDL environment, includes Hanning smoothing, bandpass subtraction, excision of radio frequency interference (RFI), and flux calibration. The spectra obtained from each on/off pair are weighted by $1 / \mathrm{rms}^{2}$, where rms is the root mean square noise measured in the signal-free portion of the spectrum, and co-added. The two orthogonal linear polarizations (kept separated up to this point) are averaged to produce the final spectrum; polarization mismatch, if significant, is noted in Appendix B. The spectrum is then boxcar smoothed, baseline subtracted and measured as explained in Catinella et al. (2010). The instrumental broadening correction for the velocity widths is described in Catinella et al. (2012b, see also Catinella et al. 2012a). Our RFI excision technique is illustrated in detail in Catinella \& Cortese (2015).

\subsubsection{The new data release}

This data release includes new Arecibo observations of 208 galaxies. The catalogues of optical, UV, and $21 \mathrm{~cm}$ parameters for these objects are presented in Appendix A.
All the optical parameters were obtained from the SDSS DR7 data base server. ${ }^{2}$ Stellar masses are from the Max Planck Institute for Astrophysics (MPA)/Johns Hopkins University (JHU) value-added catalogue based on SDSS DR7, ${ }^{3}$ and assume a Chabrier (2003) initial mass function.

UV photometry and star formation rate (SFR) measurements were obtained for the full xGASS sample as explained in detail by Janowiecki et al. (2017). Briefly, NUV magnitudes are typically from the GALEX Unique Source Catalogs ${ }^{4}$ (Seibert et al. 2012), or other GALEX catalogues such as BCSCAT (Bianchi, Conti \& Shiao 2014) and the GR6+7 data release. ${ }^{5}$ The measured NUV $-r$ colours are corrected for Galactic extinction following Wyder et al. (2007), from which we obtained $A_{N U V}-A_{r}=1.9807 A_{r}$ (where the extinction $A_{r}$ is available from the SDSS data base and reported in Table A1 below). We did not apply internal dust attenuation corrections.

SFRs were computed combining NUV with mid-infrared (MIR) fluxes from the Wide-field Infrared Survey Explorer (WISE, Wright et al. 2010). We performed our own aperture photometry on the WISE atlas images and used the $w 4$-band $(22 \mu \mathrm{m})$ measurements when possible, and $w 3$-band $(12 \mu \mathrm{m})$ ones otherwise. If good NUV and MIR fluxes were both not available, we used SFRs determined from the spectral energy distribution fits of Wang et al. (2011); we refer the reader to Janowiecki et al. (2017) for further details.

The catalogues presented in our three GASS data releases (Catinella et al. 2010, 2012b, 2013) and in this work, as well as the full xGASS representative sample (see Section 2.2), are available on the xGASS website, ${ }^{6}$ along with all the Arecibo H I spectra in digital format.

\subsection{The xGASS representative sample}

In order to increase survey efficiency we did not reobserve galaxies with good quality Hi detections in ALFALFA (based on the most recent data release available at the time of our observations, which was AA40 for GASS-low) or the Cornell $\mathrm{H}_{\text {I }}$ digital archive (S05). For ALFALFA, this refers to galaxies with detection code ' 1 ' (i.e. signal-to-noise $\mathrm{SNR} \geq 6.5$ ); sources identified by code ' 2 ' (with lower SNR but coincident with an optical counterpart at the same redshift) were reobserved. Hence both GASS and GASS-low observed samples lack $\mathrm{H}$ I-rich objects, which must be added back in the correct proportions to obtain data sets that are representative in terms of H I properties. Because the two surveys cover different volumes and stellar mass regimes, we generate the two representative samples separately, taking advantage in both cases of the more recent 70 per cent data release ${ }^{7}$ of ALFALFA (AA70). This is done slightly differently to the three GASS data releases. We explain below the procedure used to generate the xGASS representative sample, which is simply obtained by joining the GASS-low and (revised) GASS ones.

First, we divide each sample into two parts: inside and outside the AA70 footprint (see Fig. 1), which is given by the sky distribution of the $23881 \mathrm{HI}$-detected galaxies included in the publicly

\footnotetext{
${ }^{2}$ http://cas.sdss.org/dr7/en/tools/search/sql.asp

${ }^{3}$ http://www.mpa-garching.mpg.de/SDSS/DR7/; we used the improved stellar masses from http://home.strw.leidenuniv.nl/ jarle/SDSS/

${ }^{4}$ http://archive.stsci.edu/prepds/gcat/

${ }^{5} \mathrm{http} / / /$ galex.stsci.edu/GR6/

${ }^{6} \mathrm{http}: / / \mathrm{xgass}$.icrar.org

${ }^{7}$ Obtained from http://egg.astro.cornell.edu/alfalfa/data/index.php
} 

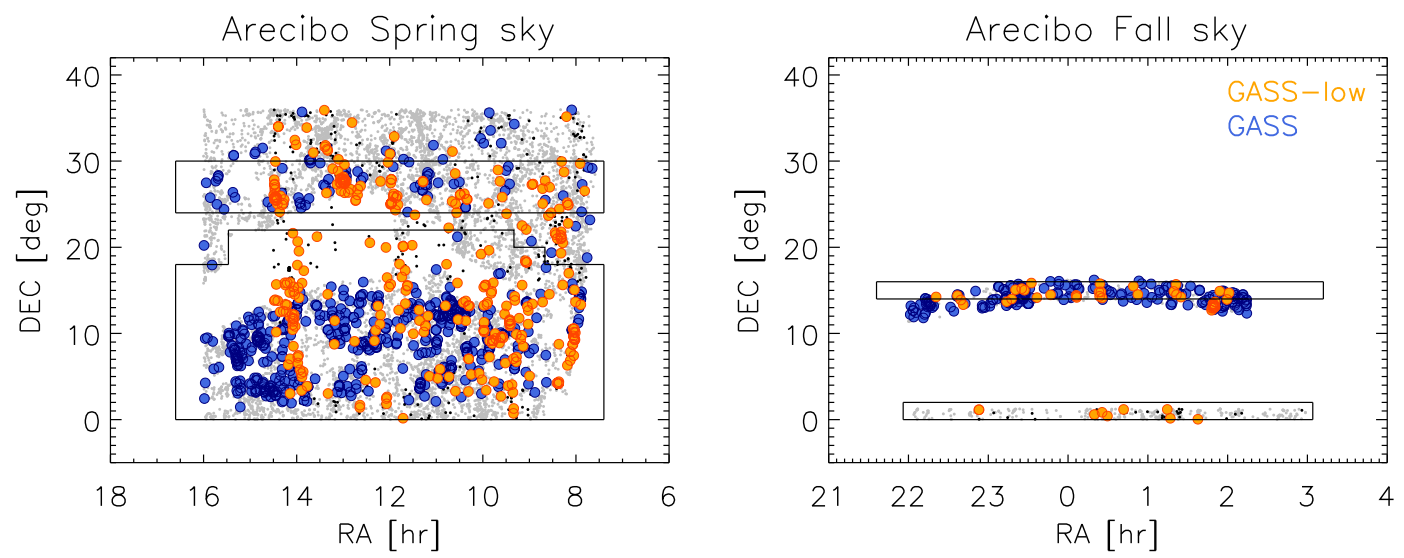

Figure 1. Sky distribution of galaxies in the GASS (blue) and GASS-low (orange) representative samples. Black and grey dots show the parent samples of the two surveys (GASS-low and GASS, respectively). The areas enclosed by thin black lines indicate the ALFALFA 70 per cent footprint in the regions of interest.

available catalogue. The fraction of GASS-low and GASS parent samples included by this footprint are 74 per cent and 75 percent, respectively. We compute the ALFALFA detection fraction in each stellar mass bin, $f_{\mathrm{AA}}$, defined as the ratio of number of galaxies $N_{\mathrm{AA}}$ detected by ALFALFA (code ' 1 ' only) and total number of sources $N_{\text {PS }}$ in the parent sample, both restricted to the sky region with complete ALFALFA coverage and to the given stellar mass bin. Detection fractions decrease from 56.5 per cent to 50.4 per cent for GASS-low and from 16.2 per cent to 13.9 per cent for GASS going from the lowest to the highest stellar mass bin.

Secondly, we generate the representative sample for the subset within the AA70 footprint by adding the correct proportion of gasrich, ALFALFA detections in each stellar mass bin. If $N_{\text {poor }}$ is the number of observed galaxies in the given stellar mass bin that are not detected by ALFALFA, we obtain the number of gas-rich galaxies to be added as follows:

$N_{\text {rich }}=N_{\text {poor }} \times \frac{N_{\mathrm{AA}} / N_{\mathrm{PS}}}{1-N_{\mathrm{AA}} / N_{\mathrm{PS}}}=N_{\text {poor }} \times \frac{f_{\mathrm{AA}}}{1-f_{\mathrm{AA}}}$,

where $N_{\mathrm{PS}}$ is the number of galaxies in the parent sample within the AA70 footprint in the given stellar mass bin. We denote as $N_{\text {xGASS, rich }}$ those galaxies that we observed but are also ALFALFA detections (for instance, GASS galaxies outside the AA40 footprint that turned out to be detected in AA70). These galaxies are not included in $N_{\text {poor }}$, but we pick them first as gas-rich systems to be added to the sample. Next, we select $N_{\text {rich }}-N_{\text {xGASS, rich }}$ uniformly distributed, random galaxies from ALFALFA (giving first preference to sources with xCOLD GASS data) and we add them to $N_{\text {poor }}+N_{\mathrm{xGASS} \text {, rich }}$ to obtain our representative sample.

Thirdly, we deal with the part of the sample outside the AA70 footprint. Here, we need to estimate which galaxies would be detected by ALFALFA if the survey was complete. ${ }^{8}$ Because the sensitivity (or completeness) of ALFALFA depends on both flux and velocity width of the H I signal (see Section 6 of Haynes et al. 2011), we inspect the histogram of the $\mathrm{H}_{\mathrm{I}}$ integrated flux, $F_{\mathrm{HI}}$, divided by the observed velocity width, $W_{50}^{c}$ (measured at the 50 per cent peak

\footnotetext{
${ }^{8}$ This step is necessary for GASS-low due to the inclusion of S05 galaxies, and we wanted to treat these and our Arecibo observations in a uniform way. Furthermore, GASS lacks $\mathrm{H}$ I-rich galaxies at high stellar masses outside the AA70 footprint, most likely due to a combination of large-scale structure and Arecibo time allocation, so our procedure corrects for this. However, as already noted, both GASS and GASS-low samples are dominated by the subsets within the AA70 footprint.
}

level and corrected for instrumental broadening and redshift only, see Appendix A) to decide where to set the threshold. Fig. 2 shows the $F_{\mathrm{HI}} / W_{50}^{c}$ histograms for GASS-low (left) and GASS (right) galaxies, compared with ALFALFA detections within the corresponding parent samples (codes ' 1 ' and ' 2 ' are indicated in light blue and green, respectively). For both surveys we adopt a value of $0.005 \mathrm{Jy}$ as our threshold (dotted lines), below which ALFALFA code '2' sources start dominating over high signal-to-noise ones. We verified that changing this number slightly does not have a significant effect on the final sample (changing the sky footprint over which detection fractions are computed, e.g. from AA40 to AA70, has a much larger effect). Then we use equation (1) to generate our representative samples, where $N_{\text {poor }}$ now includes xGASS galaxies with $F_{\mathrm{HI}} / W_{50}^{c}<0.005 \mathrm{Jy}$ (i.e. below the ALFALFA detection limit) and $N_{\mathrm{xGASS}}$, rich those above this threshold. The $\mathrm{H}$ I-rich galaxies are extracted randomly from ALFALFA detections not already in the sample, trying to maximize the overlap with xCOLD GASS.

In our GASS papers, we treated the $\mathrm{S} 05 \mathrm{HI}$ archive in a similar way as ALFALFA: we computed the fraction of parent sample galaxies with $\mathrm{H}_{\mathrm{I}}$ data in the archive, $f_{\mathrm{S} 05}$ (not including ALFALFA detections), and used equation (1) (with $f_{\mathrm{S} 05}$ replacing $f_{\mathrm{AA}}$ ) to obtain the number of $\mathrm{H}$ I-rich $\mathrm{S} 05$ galaxies to be added to the observed sample. While this does not affect our scaling relations (only 1.3 per cent of the galaxies in the GASS DR3 representative sample were from $\mathrm{S} 05^{9}$ ), this is not entirely correct, because the S05 archive is not an $\mathrm{H}$ I-blind survey, and thus $f_{\mathrm{S} 05}$ is not a meaningful detection fraction. Thus, we no longer add $\mathrm{H}$ I-rich S05 galaxies to the observed sample.

We also considered including S05 galaxies below the ALFALFA detection threshold, together with the right complement of ALFALFA detections, to increase our statistics. This cannot be done in the GASS volume, where we would add 104 'H I-poor' S05 galaxies, because the H I archive sample is deeper than ALFALFA but still H I-rich compared to GASS (thus we would bias our sample). However, this is not the case for the GASS-low volume, where there

\footnotetext{
${ }^{9}$ Contrary to what we did for GASS in our previous papers, we now use ALFALFA instead of S05 H I fluxes for galaxies detected in both catalogues. While S05 integration times are typically longer, the spectra were obtained with a variety of single-dish radio telescopes, hence have variable sensitivity, spatial and spectral resolutions. Thus, 36 out of 760 galaxies in the GASS DR3 representative sample had $\mathrm{H}$ I measurements from S05, but only 10 of these are not detected by ALFALFA.
} 

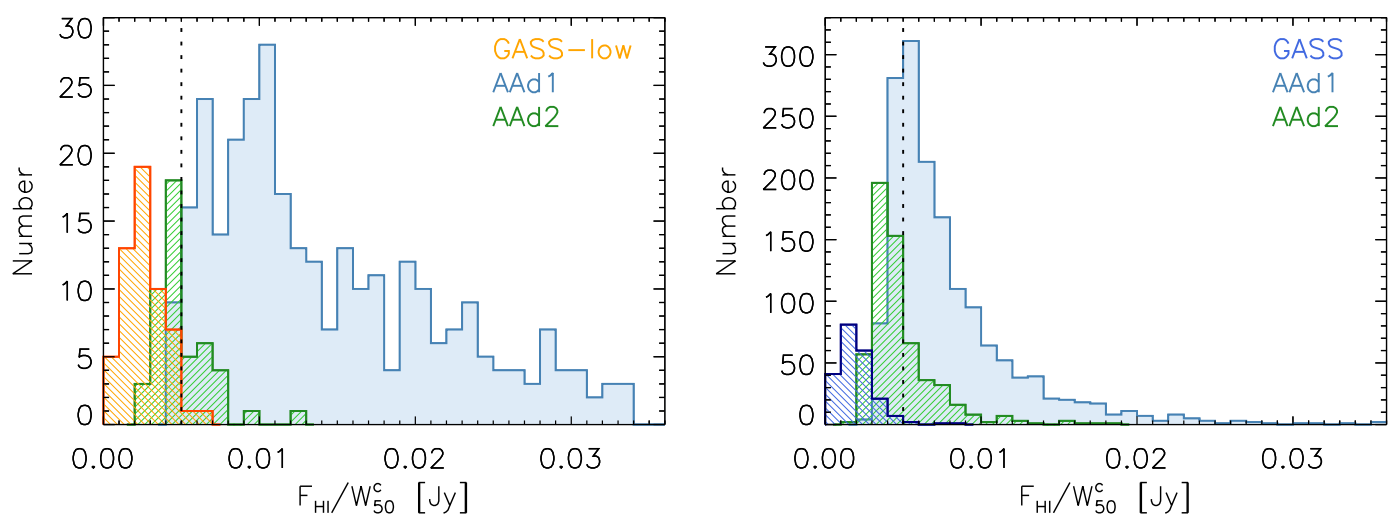

Figure 2. Histograms of the mean flux density across the detected $\mathrm{H}_{\mathrm{I}}$ signal for GASS-low (left) and GASS (right) surveys, with ALFALFA detections (quality codes 1 and 2) in the corresponding volumes. Dotted lines indicate the value adopted to separate gas-rich from gas-poor galaxies in regions of sky not covered by ALFALFA (see the text).
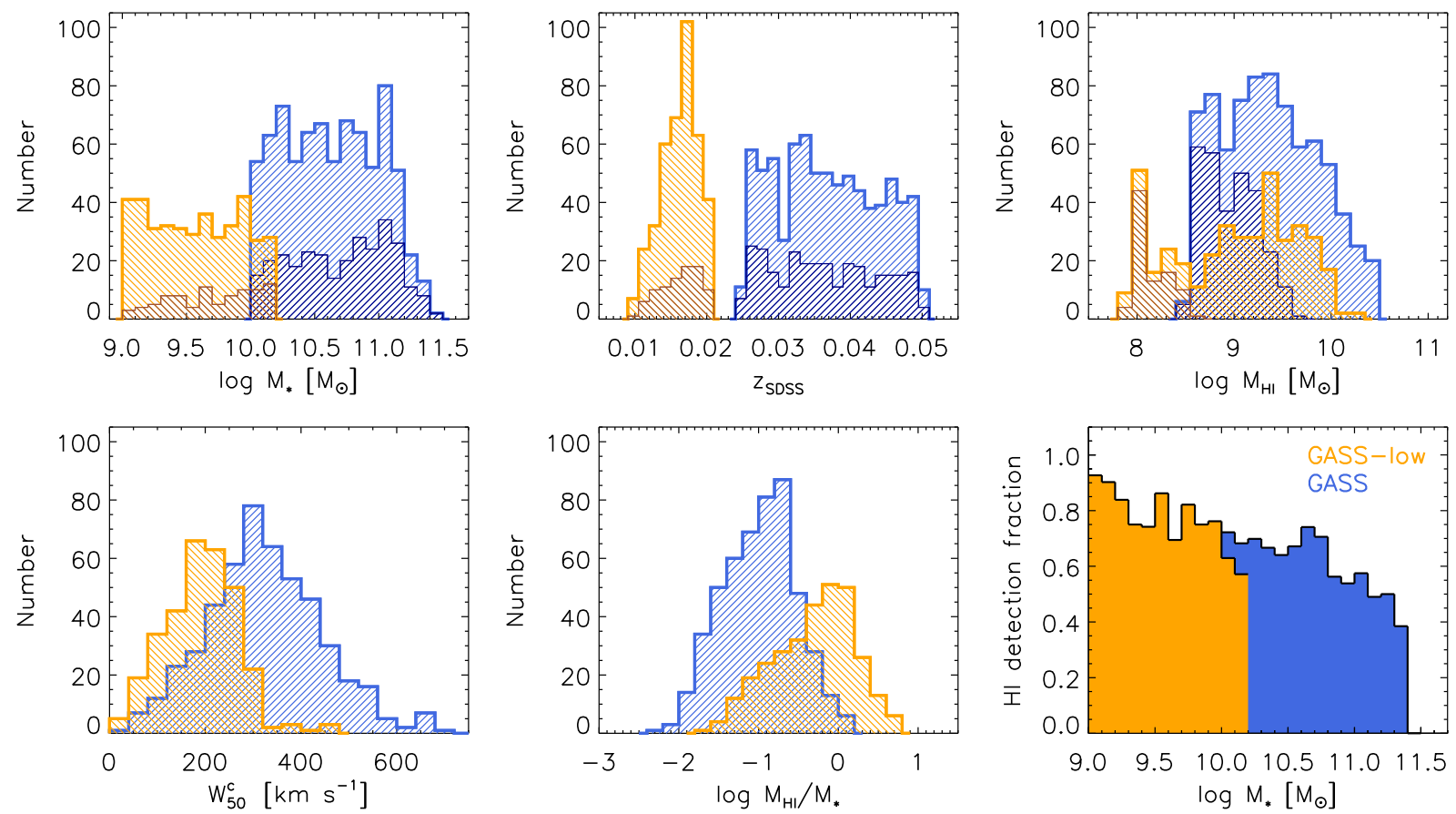

Figure 3. xGASS representative sample properties. Top row: distributions of stellar mass, optical redshift, and H mass for GASS (blue) and GASS-low (orange); dark blue and brown histograms indicate $\mathrm{H}$ I non-detections for the two surveys, respectively. Bottom row: distributions of $\mathrm{H}$ I velocity width (corrected for instrumental broadening and redshift) and $\mathrm{H}_{\mathrm{I}}$ gas fraction for detected galaxies; the Hi detection fraction (i.e. the ratio of detections to total) as a function of stellar mass is shown on the right-hand panel (blue: GASS; orange: GASS-low).

are only 13 'H I-poor' S05 galaxies, all with gas fractions comparable to our observations. Thus, we include these in our sample as if they had been observed by us (i.e. increasing $N_{\text {poor }}$ in equation 1), and verified that our scaling relations are not affected by this choice.

The new GASS and GASS-low representative samples include 781 and 398 galaxies, respectively, for a total 1179 xGASS galaxies. Fig. 3 summarizes the main properties of the sample. On the top row, the distributions of stellar mass, optical redshift from SDSS and $\mathrm{H}_{\mathrm{I}}$ mass are shown separately for GASS (blue) and GASS-low (orange); non-detections are indicated in dark blue and brown, respectively. On the bottom row, we show the histograms of velocity width and gas fraction for the $\mathrm{H}$ I detections, as well as the $\mathrm{H}$ I detection fraction as a function of stellar mass for the two surveys. The $\sim 10$ per cent detection rate difference in the two overlapping stellar mass bins is most likely just noise (a couple more detections in GASS-low would have brought the detection rates into agreement). The observed velocity widths peak at $\sim 200$ and $300 \mathrm{~km} \mathrm{~s}^{-1}$ for GASS-low and GASS, respectively, so these are the values that we adopt to compute upper limits for the $\mathrm{H}$ I mass of non-detections in the two surveys.

\subsubsection{Recovering a volume-limited sample}

In our previous GASS work, we computed average scaling relations by weighting each measured gas fraction (detection or upper limit) by a factor $w_{i}\left(\mathrm{M}_{\star}\right)$, in order to compensate for the flat stellar mass distribution imposed on the survey. Weights were computed using 


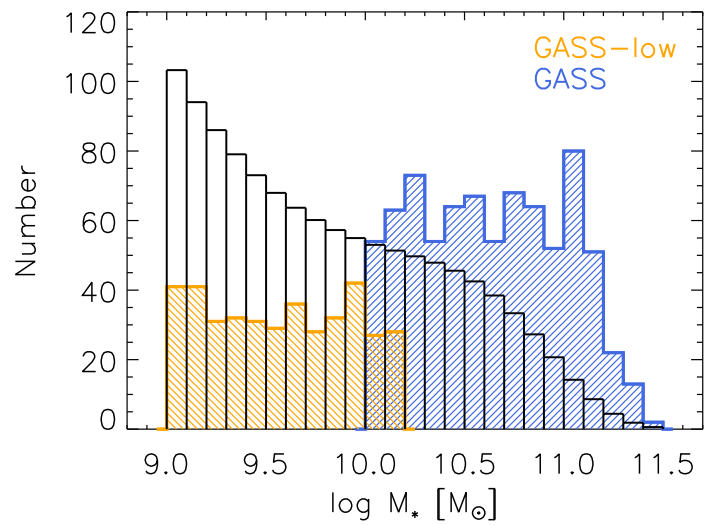

Figure 4. Stellar mass distributions for the xGASS representative sample (orange and blue indicate GASS-low and GASS subsets, respectively) and for a volume-limited sample with the same number of galaxies (see the text).

the parent sample as a reference, by binning both parent and representative samples by stellar mass and taking the ratio between the two histograms.

The xGASS representative sample has a similar problem, largely due to the difference in sample size between GASS and its low-mass extension - low-mass galaxies are under-represented and high-mass ones over-represented, compared to what is expected for a volumelimited sample. This is illustrated in Fig. 4, which shows the stellar mass distributions for GASS-low (orange histogram) and GASS (blue) representative samples, and for a volume-limited sample with the same total number of galaxies (black). The latter was obtained by sampling the local stellar mass function, parametrized as a double Schechter function ${ }^{10}$ by Baldry et al. (2012):

$\Phi(\log M) \mathrm{d} \log M=\ln (10) \mathrm{e}^{-x}\left[\phi_{1}^{*} x^{\left(1+\alpha_{1}\right)}+\phi_{2}^{*} x^{\left(1+\alpha_{2}\right)}\right] \mathrm{d} \log M$,

where $x=10^{\log M-\log M^{*}}, \log M^{*}\left[\mathrm{M}_{\odot}\right]=10.66, \phi_{1}^{*}=0.00396$, $\phi_{2}^{*}=0.00079, \alpha_{1}=-0.35$, and $\alpha_{2}=-1.47$.

Thus, in this work we use the above stellar mass function to weight the gas fraction measurements when we compute average and median gas scaling relations; weights are simply obtained as the ratio between the black and the coloured histograms in Fig. 4 for the corresponding stellar mass bin and survey (GASS or GASS-low).

\section{XCOLD GASS: THE MOLECULAR GAS SURVEY}

The COLD GASS survey measured homogeneous molecular gas masses, via the $\mathrm{CO}(1-0)$ emission line fluxes, for 366 galaxies extracted from the GASS sample (Saintonge et al. 2011). Because the IRAM beam size at the frequency of the observed $\mathrm{CO}(1-0)$ transition is 22 arcsec, aperture corrections were applied to extrapolate the measured CO line fluxes to total fluxes, as described in Saintonge et al. (2012). The extension of COLD GASS to a stellar mass of $10^{9} \mathrm{M}_{\odot}$, COLD GASS-low, includes IRAM observations of 166 additional galaxies, randomly extracted from the GASS-low parent sample (see Section 2.1.1). The two surveys taken together constitute $x$ COLD GASS, which includes 532 galaxies (333 detections) and is described in detail in Saintonge et al. (2017).

The $\mathrm{CO}(1-0)$ fluxes are converted into $\mathrm{H}_{2}$ molecular masses using a multivariate conversion function, $\alpha_{\mathrm{CO}}$, following Accurso et al.

${ }^{10}$ We use the logarithmic form from Moffett et al. (2016).
(2017). This function depends primarily on metallicity and secondarily on the offset from the star-forming main sequence (SFMS), i.e. a parameter related to the strength of the UV radiation field; $\alpha_{\mathrm{CO}}$ values for $x$ COLD GASS detections vary between 1 and $24.5 \mathrm{M}_{\odot}(\mathrm{K}$ $\left.\mathrm{km} \mathrm{s}^{-1} \mathrm{pc}^{2}\right)^{-1}$, with an average of 4.44 (for comparison, the Galactic value is 4.35), taking into account the contribution of helium. In this work, we use molecular gas masses without helium contribution, $M_{\mathrm{H}_{2}}$, to compute molecular-to-atomic hydrogen gas mass ratios.

The overlap between xCOLD GASS and the xGASS representative sample, which we refer to as $x G A S S-C O$, includes 477 galaxies (290 CO detections) and is used in this work to investigate total gas scaling relations and $\mathrm{H}_{2} / \mathrm{H}$ I mass ratios. The remaining 55 galaxies with CO data are not included in xGASS because of one of the following reasons: (a) lack of $\mathrm{H}$ I observations (13); (b) specifically targeted by COLD GASS for their very high specific SFRs, hence not preferentially selected for our representative sample $(35,2$ of which were randomly picked as ALFALFA 'code 1' sources); (c) S05 detections in GASS (7); or (d) ALFALFA 'code 1' sources that were not selected because the stellar mass bin already included enough $\mathrm{H}$ I-rich systems (2).

We recomputed the weights for xGASS-CO in order to recover the stellar mass distribution of a volume-limited sample, following the procedure described in Section 2.2.1. This sample is representative in terms of $\mathrm{H}$ I content (we verified that the average $\mathrm{H}_{\mathrm{I}}$ scaling relations obtained for $\mathrm{xGASS}$ and for xGASS-CO are consistent within the errors).

\section{RESULTS}

We briefly revisit the main $\mathrm{H}_{\mathrm{I}}$ gas fraction scaling relations, which extend our previous work (Catinella et al. 2010, 2012b, 2013) to lower stellar masses, and take advantage of the combined $\mathrm{H}_{\mathrm{I}}$ and $\mathrm{H}_{2}$ data set to investigate total gas scaling relations. Molecular gas scaling relations are presented in a companion paper (Saintonge et al. 2017). As discussed below, the distinct behaviour of the atomic and molecular phases at low stellar masses motivates a more detailed discussion of the molecular-to-atomic gas mass ratio of galaxies along and outside the star-forming sequence.

\subsection{Atomic gas fraction scaling relations}

The H I gas fraction scaling relations are presented in Fig. 5. Clockwise from the top left, we show how the gas mass fraction $M_{\mathrm{HI}} / M_{\star}$ varies with stellar mass, stellar mass surface density, observed NUV $-r$ colour and specific SFR (sSFR) for the full xGASS representative sample. Circles and downward arrows indicate $\mathrm{H}$ I detections and $\mathrm{H}$ I upper limits, respectively, with the new GASS-low survey shown in orange. The low-mass galaxies smoothly extend GASS trends by 1 dex in stellar mass, probing higher gas fractions and sSFRs, bluer colours and lower stellar surface densities typical of disc-dominated systems. Because some of these relations do not appear linear (especially those with colour and sSFR), we quantified their strength with the Spearman's rank correlation coefficient, $r_{\mathrm{s}}$, computed including the upper limits. The most significant correlation is with NUV $-r$ colour $\left(r_{\mathrm{s}}=-0.82\right)$, with the $M_{\star}$ and $\mu_{\star}$ relations having significantly lower correlation coefficients $\left(r_{\mathrm{s}}=-0.59\right.$ and -0.69 , respectively).

This is better seen in Fig. 6, which quantifies the observed trends in terms of average (large red circles) and median (blue diamonds) gas fractions. The values plotted are weighted means and weighted 

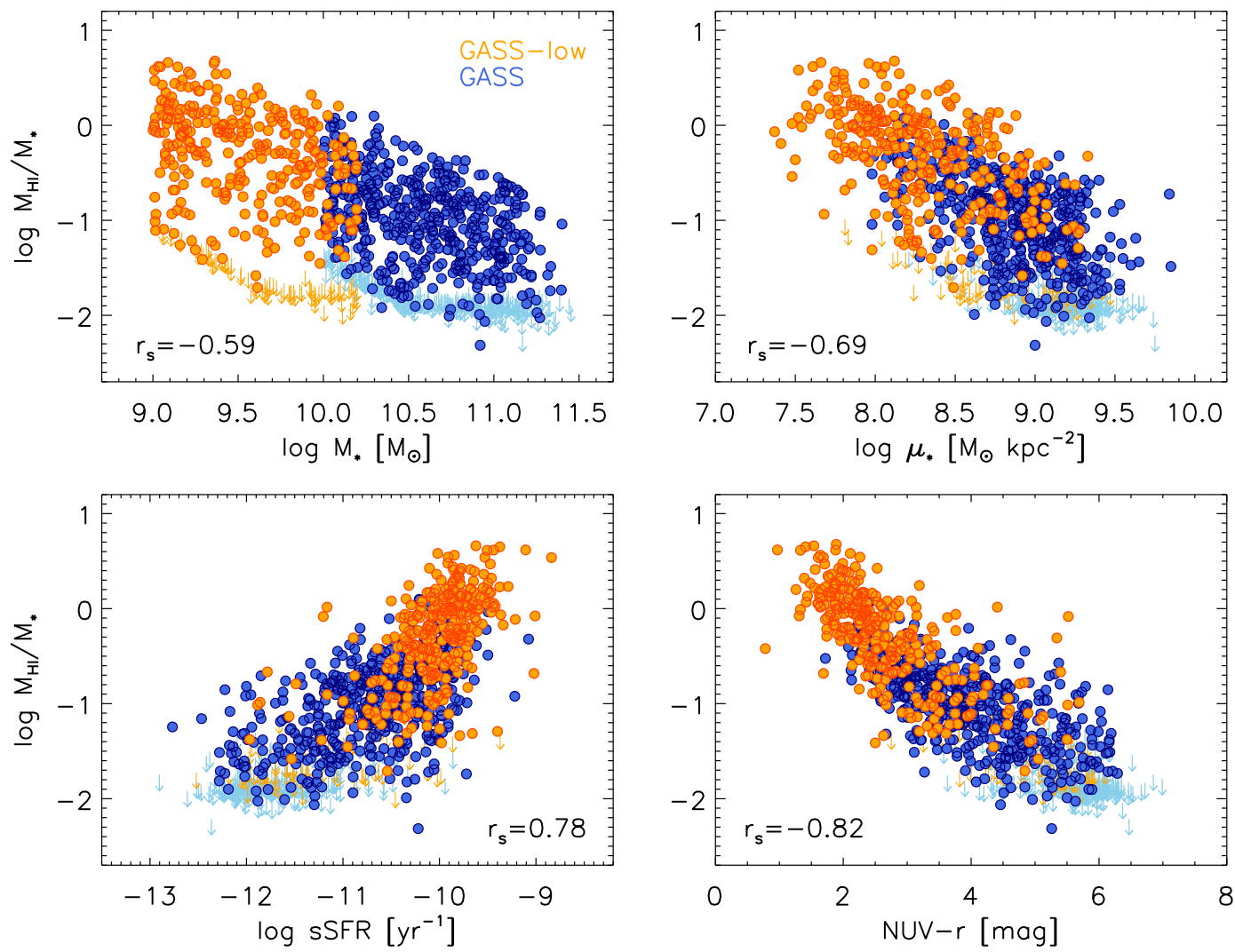

Figure 5. xGASS scaling relations: the $\mathrm{H}_{\mathrm{I}}$ mass fraction is plotted as a function of stellar mass, stellar mass surface density, specific SFR and observed NUV $-r$ colour. Circles and downward arrows indicate individual $\mathrm{H}$ I detections and $5 \sigma$ upper limits, respectively, with the new GASS-low observations shown in orange. The Spearman's correlation coefficient, $r_{\mathrm{s}}$, is given on the bottom of each panel.

medians ${ }^{11}$ of the logarithm of the gas fractions, where the weighting is applied to correct for the stellar mass bias of the sample (see Section 2.2.1); non-detections were set to their upper limits. For reference, small grey circles and downward arrows reproduce individual $\mathrm{H}$ I detections and upper limits from Fig. 5. Average and median gas fractions track each other closely in all plots, despite the fact that the underlying distributions are clearly not lognormal; however, medians are preferable descriptors because less sensitive to the treatment of non-detections, which could lie anywhere below the upper limits. The values of the weighted average and median gas fractions shown in this figure are listed in Table 1 . In order to quantify the scatter in these relations, we computed the difference between the 75th and 25th percentiles of the $\log \left(M_{\mathrm{HI}} / M_{\star}\right)$ distributions in each bin $\left(\Delta_{75-25, i}\right.$, including the $\mathrm{H}_{\mathrm{I}}$ non-detections at their upper limits), and took their arithmetic mean $(\bar{\Delta})$. We obtained $\bar{\Delta}=0.96,0.73,0.51$ and 0.43 dex for the $M_{\star}, \mu_{\star}$, SSFR and NUV $-r$ relations, respectively, with no clear trends in $\Delta_{75-25, i}$ with any of the above quantities (except for an artificially lower scatter in the two bins dominated by non-detections in each relation).

Figs 5 and 6 show that $\mathrm{H}$ gas fraction is more tightly related to NUV $-r$ colour and sSFR, and both relations steepen in the star-forming sequence (approximately corresponding to NUV $-r$

\footnotetext{
${ }^{11}$ Given $n$ elements $x_{1} \ldots x_{n}$ with positive weights $w_{1} \ldots w_{n}$ such that their sum is 1 , the weighted median is defined as the element $x_{k}$ for which: $\sum_{x_{i}<x_{k}} w_{i}<1 / 2$ and $\sum_{x_{i}>x_{k}} w_{i} \leq 1 / 2$.
}

$<3$ mag and $\left.\log \mathrm{sSFR}\left[\mathrm{yr}^{-1}\right]>-10.5\right)$. This change of slope could be due to a saturation effect at the opposite end, where we hit the survey sensitivity limit and upper limits dominate the statistics. Contrary to the molecular gas fraction, which correlates more strongly with sSFR (Saintonge et al. 2017), the atomic gas fraction is more tightly related to NUV $-r$ colour $(\bar{\Delta}=0.43 \mathrm{dex})$, which traces dust-unobscured star formation (Bigiel et al. 2010).

H I gas fractions keep increasing with decreasing stellar mass, with no sign of a plateau, down to $M_{\star}=10^{9} \mathrm{M}_{\odot}$ (median values of $M_{\mathrm{HI}} / M_{\star}$ increase from 2 per cent to 81 per cent from the highest to the lowest stellar mass bin). This is consistent with the relation for H I-rich galaxies detected by ALFALFA, which shows a flattening only below $M_{\star} \sim 10^{8.5} \mathrm{M}_{\odot}$ (Huang et al. 2012). The correlation between $\mathrm{H}_{\mathrm{I}}$ gas fraction and stellar mass has the largest scatter ( $\bar{\Delta}=0.96 \mathrm{dex}$ ). This is not surprising, as we already showed in previous work that variations of atomic gas fraction at fixed stellar mass strongly correlate with star formation activity (Brown et al. 2015). By applying spectral stacking to a large stellar mass-selected sample with H I data from ALFALFA, Brown et al. (2015, see their fig. 5) demonstrated that this relation is the result of a more physical correlation between $\mathrm{H}$ I content and SFR, combined with the bimodality of galaxies - dividing up their sample in three NUV $-r$ colour bins, roughly corresponding to blue sequence, red sequence, and green valley, they obtained three parallel relations with significantly flatter slope.

Interestingly, the relation with stellar surface density has lower scatter $(\bar{\Delta}=0.73 \mathrm{dex})$, indicating that the correlation between gas fraction and stellar content improves when we take into account the size of the stellar disc (even if estimated as a 50 per cent effective 

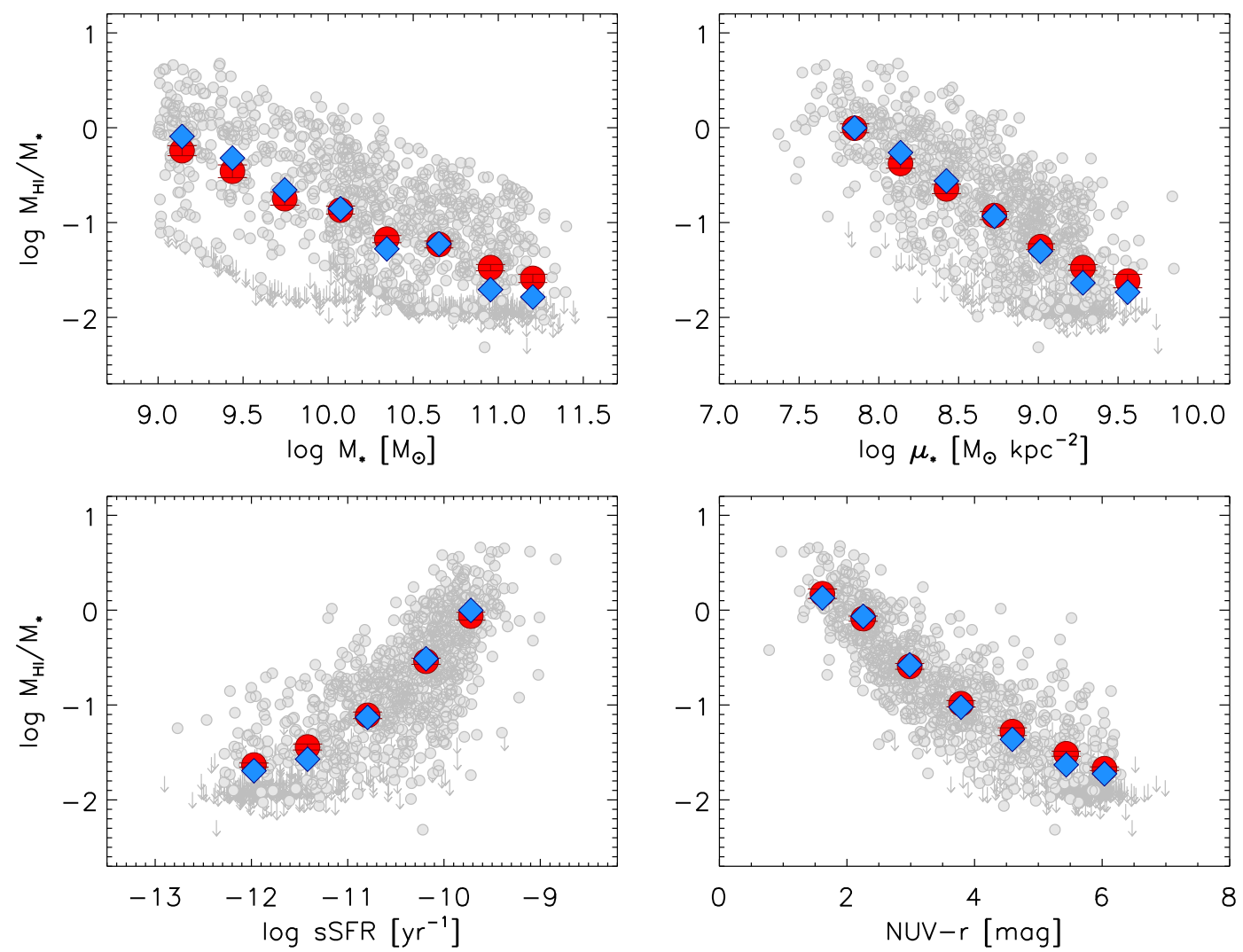

Figure 6. Average trends of $\mathrm{H}_{\mathrm{I}}$ mass fraction as a function of stellar mass, stellar mass surface density, specific SFR, and observed NUV- $r$ colour for the xGASS sample. In each panel, large red circles and blue diamonds indicate weighted averages and weighted medians of the logarithms of the gas fractions, respectively (see the text). Only bins including at least 20 galaxies are shown. Error bars are errors on the weighted means. These results are listed in Table 1. Small circles and downward arrows show individual $\mathrm{H}_{\mathrm{I}}$ detections and non-detections (plotted at their upper limits), respectively.

radius). As noted before, a distinct difference between the $M_{\star}$ and $\mu_{\star}$ relations is the distribution of the non-detections, which are spread across the stellar mass range but pile up in the bulge-dominated region $\left(\log \mu_{\star}\left[\mathrm{M}_{\odot} \mathrm{kpc}^{-2}\right] \gtrsim 8.5\right)-$ above this threshold, both $\mathrm{HI}$ and $\mathrm{H}_{2}$ detection rates drop significantly (Catinella et al. 2010; Saintonge et al. 2011). Galaxies in the lowest stellar surface density bin have median gas fractions of 100 per cent, i.e. have the same amount of mass in $\mathrm{H}$ I gas and in stars; those with the bluest NUV $-r$ colours are gas-dominated, reaching median gas fractions of 135 per cent.

\subsection{Total gas fraction scaling relations}

In the rest of this paper we restrict our analysis to the subset of xGASS with IRAM observations, xGASS-CO, which includes 477 galaxies (see Section 3).

Fig. 7 shows the distribution of this sample in the specific SFR versus stellar mass plane, with points colour-coded according to their detection status in the $\mathrm{H}_{\mathrm{I}}$ and $\mathrm{H}_{2}$ surveys. About 57 percent of the sample is detected in both lines (filled, dark blue circles), 16 per cent is detected only in $\mathrm{H}_{\mathrm{I}}$ (light blue) and 23 per cent has no cold gas detection (crosses). As expected, galaxies on or above the SFMS (dotted lines; see below) are typically $\mathrm{H}_{\mathrm{I}}$ and $\mathrm{H}_{2}$ detections, except at the low stellar mass end, where metallicities are lower and $\mathrm{CO}$ emission is more challenging to detect. We marked with black-edged diamonds 39 galaxies ( 8 per cent of xGASS-CO) with $\mathrm{H}$ I emission that is confused within the Arecibo beam (see the appendices); together with the non-detections in both gas phases, these objects are excluded from the analysis of molecular-to-atomic gas mass ratios in the next section. It is interesting to note that 17 galaxies (4 per cent of our sample, empty circles) are detected in $\mathrm{CO}$ only; of these, 9 are satellites in groups with 19 or more members according to the Yang et al. (2007) group catalogue ${ }^{12}$ and are all located in the bottom half of the SFMS or below it, suggesting that environmental effects in these large groups might have depleted the $\mathrm{H}$ I reservoirs, but not as far inside as the optical disc, thus leaving the $\mathrm{H}_{2}$ content mostly unaffected (Fumagalli et al. 2009; Boselli et al. 2014b; Cortese et al. 2016).

We defined our own SFMS using the full xGASS representative sample. Briefly, we binned the points in the sSFR-stellar mass plot (not shown) in stellar mass intervals of 0.25 dex, and fit Gaussians to the resulting sSFR distributions; this works well below $M_{\star} \sim 10^{10}$ $\mathrm{M}_{\odot}$, where the red sequence is almost absent. At higher stellar masses, we fit Gaussians with fixed centres based on the extrapolation of the relation at lower $M_{\star}$, and use only sSFRs above the relation to constrain the widths of the Gaussians. This procedure (illustrated in more detail in Janowiecki et al., in preparation) yields the following expression:

$\log \mathrm{SSFR}_{\mathrm{MS}}=-0.344\left(\log M_{\star}-9\right)-9.822$,

with a standard deviation given by

$\sigma_{\mathrm{MS}}=0.088\left(\log M_{\star}-9\right)+0.188$.

${ }^{12}$ We use their SDSS DR7 'B' catalogue, available online at http://gax.shao.ac.cn/data/Group.html; see Janowiecki et al. (2017) for more details. 
Table 1. H I gas fraction scaling relations for xGASS.

\begin{tabular}{|c|c|c|c|c|}
\hline$x$ & $\langle x\rangle$ & $\left\langle M_{\mathrm{HI}} / M_{\star}\right\rangle^{a}$ & $\left(M_{\mathrm{HI}} / M_{\star}\right)^{b}$ & $N^{c}$ \\
\hline \multirow[t]{8}{*}{$\log M_{\star}$} & 9.14 & $-0.242 \pm 0.053$ & -0.092 & 113 \\
\hline & 9.44 & $-0.459 \pm 0.067$ & -0.320 & 92 \\
\hline & 9.74 & $-0.748 \pm 0.069$ & -0.656 & 96 \\
\hline & 10.07 & $-0.869 \pm 0.042$ & -0.854 & 214 \\
\hline & 10.34 & $-1.175 \pm 0.037$ & -1.278 & 191 \\
\hline & 10.65 & $-1.231 \pm 0.036$ & -1.223 & 189 \\
\hline & 10.95 & $-1.475 \pm 0.033$ & -1.707 & 196 \\
\hline & 11.20 & $-1.589 \pm 0.044$ & -1.785 & 86 \\
\hline \multirow[t]{7}{*}{$\log \mu_{\star}$} & 7.85 & $-0.006 \pm 0.047$ & -0.002 & 61 \\
\hline & 8.14 & $-0.377 \pm 0.050$ & -0.262 & 129 \\
\hline & 8.42 & $-0.646 \pm 0.049$ & -0.561 & 160 \\
\hline & 8.72 & $-0.926 \pm 0.044$ & -0.934 & 221 \\
\hline & 9.01 & $-1.255 \pm 0.032$ & -1.303 & 326 \\
\hline & 9.28 & $-1.475 \pm 0.031$ & -1.636 & 233 \\
\hline & 9.56 & $-1.617 \pm 0.071$ & -1.734 & 30 \\
\hline \multirow[t]{5}{*}{$\log$ SSFR } & -11.97 & $-1.633 \pm 0.022$ & -1.694 & 204 \\
\hline & -11.42 & $-1.442 \pm 0.032$ & -1.571 & 214 \\
\hline & -10.79 & $-1.109 \pm 0.034$ & -1.130 & 233 \\
\hline & -10.19 & $-0.539 \pm 0.033$ & -0.512 & 342 \\
\hline & -9.72 & $-0.063 \pm 0.041$ & -0.002 & 153 \\
\hline \multirow[t]{7}{*}{$\mathrm{NUV}-r$} & 1.62 & $0.174 \pm 0.050$ & 0.130 & 39 \\
\hline & 2.25 & $-0.090 \pm 0.028$ & -0.065 & 190 \\
\hline & 2.98 & $-0.593 \pm 0.030$ & -0.577 & 198 \\
\hline & 3.79 & $-0.987 \pm 0.033$ & -1.023 & 180 \\
\hline & 4.59 & $-1.281 \pm 0.040$ & -1.362 & 155 \\
\hline & 5.44 & $-1.514 \pm 0.026$ & -1.631 & 245 \\
\hline & 6.04 & $-1.672 \pm 0.020$ & -1.725 & 144 \\
\hline
\end{tabular}

Notes. ${ }^{a}$ Weighted average of logarithm of gas fraction; H I mass of nondetections set to upper limit.

${ }^{b}$ Weighted median of logarithm of gas fraction; H I mass of non-detections set to upper limit.

${ }^{c}$ Number of galaxies in the bin.

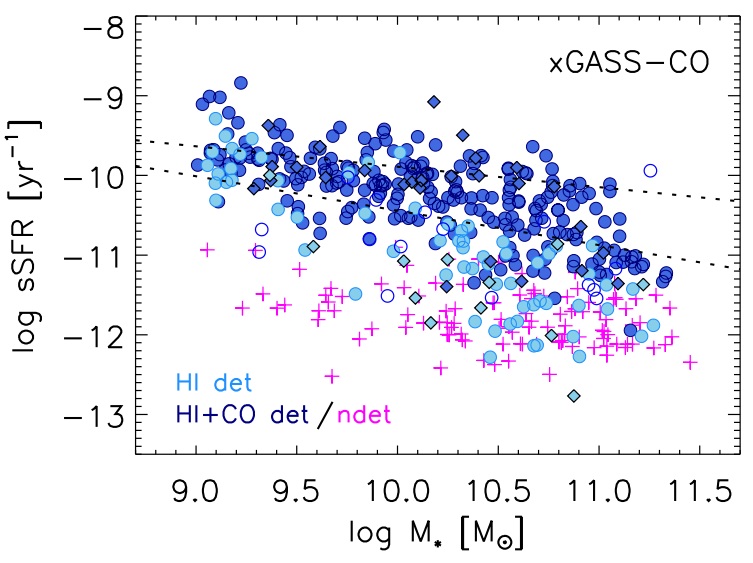

Figure 7. Specific SFR plotted as a function of stellar mass for the subset of xGASS with $\mathrm{CO}$ data. Filled circles indicate galaxies detected in both $\mathrm{H}$ I and $\mathrm{CO}$ (dark blue) or $\mathrm{H}$ I only (light blue); black-edged diamonds are $\mathrm{H}$ I detections affected by beam confusion. Empty circles are $\mathrm{CO}$ detections with $\mathrm{H}_{\mathrm{I}}$ upper limits, and magenta crosses are non-detections in both $\mathrm{HI}$ and $\mathrm{CO}$ lines. Dotted lines show the star-forming sequence adopted in this work (see the text), and correspond to the $1 \sigma$ deviation above and below the average.
The limits corresponding to $\pm 1 \sigma_{\mathrm{MS}}$ from the SFMS are shown as dotted lines in Fig. 7.

Fig. 8 shows the scaling relations for the total gas (where $M_{\text {gas }}=$ 1.3 $\left(M_{\mathrm{HI}}+M_{\mathrm{H}_{2}}\right)$, including the helium contribution). As in Fig. 6, weighted average (large red circles) and median (blue diamonds) gas fractions are plotted on top of individual measurements (small grey symbols; circles with darker contours are galaxies detected in either $\mathrm{H}_{\mathrm{I}}$ or $\mathrm{CO}$ ), with the same axis scales for comparison. Median total gas fractions (computed including all detections and upper limits) decrease from 141 per cent to 3 per cent over our stellar mass range; the galaxies with the highest total gas fractions in our sample have six times more mass in cold gas than stars.

The observed trends are qualitatively similar to the ones seen for the atomic phase, but with slightly smaller scatter, especially in the relations between gas fraction and stellar mass or stellar surface density. If we quantify the dispersions of these relations with the parameter $\bar{\Delta}$ defined in the previous section, i.e. the average difference between the 75 th and 25 th percentiles of the $\log \left(M_{\mathrm{gas}} / M_{\star}\right)$ distributions in each bin, we obtain $\bar{\Delta}=0.70,0.61,0.47$, and 0.36 dex for the $M_{\star}, \mu_{\star}, \mathrm{sSFR}$, and NUV $-r$ relations. These should be compared with the dispersions of the $\mathrm{H}$ i scaling relations computed for the same XGASS-CO sample, which are $\bar{\Delta}=0.81,0.69,0.50$, and $0.40 \mathrm{dex}$, respectively. This difference is most likely due to the fact that the total gas fractions have smaller dynamic range than the atomic ones.

Overall, the similarity between atomic and total gas scaling relations is not surprising, as galaxies in this stellar mass regime in the local Universe typically have cold gas reservoirs that are $\mathrm{HI}_{\mathrm{I}}$ dominated (Saintonge et al. 2011; Boselli et al. 2014a; Saintonge et al. 2016, see also next section).

Our total gas scaling relations confirm and extend to higher stellar mass the results of Boselli et al. (2014a), obtained for field late-type galaxies detected in both $\mathrm{H}$ r and $\mathrm{CO}$ lines in the Herschel Reference Survey (HRS; Boselli et al. 2010), assuming a luminosity-dependent $\mathrm{X}_{\mathrm{CO}}$ conversion factor to compute molecular gas masses. For reference, the HRS results are shown as black circles connected by lines in Fig. 8 (left-hand panels). The agreement with their stellar mass relation in the overlap interval $\left(\log M_{\star}\left[\mathrm{M}_{\odot}\right]<10.5\right)$ is excellent, except for their highest stellar mass bin, which has a total gas fraction 0.4 dex higher than ours, probably due to limited statistics of the HRS at the high $M_{\star}$ end. The relation with sSFR for the HRS galaxies has the same slope but is slightly offset towards lower gas fractions (by $\sim 0.2 \mathrm{dex}$ ); however, the two samples overlap by only $\sim 1.5$ dex in sSFR.

Interestingly, Boselli et al. (2014a) noted that the HRS relationships involving molecular gas fractions are always flatter than those with total gas fraction. This is confirmed by our sample (Accurso et al. 2017; Saintonge et al. 2017). Indeed, thanks to the larger dynamic range in $M_{\star}$ and $\mu_{\star}$ of XCOLD GASS, we detect a clear break in the molecular gas relations, which suddenly flatten below $\log M_{\star}\left[\mathrm{M}_{\odot}\right]=10.5$ and $\log \mu_{\star}\left[\mathrm{M}_{\odot} \mathrm{kpc}^{-2}\right]=8.5$ (see Accurso et al. 2017; Saintonge et al. 2017). As seen in Fig. 8, there is no trace left of such flattening in the total gas relations. The difference between atomic and molecular gas fraction relations below these stellar mass and stellar surface density limits is striking, and warrants a closer look at the molecular-to-atomic mass ratio in the next section.

\subsection{Molecular-to-atomic gas mass ratios}

In our previous work we investigated the relation between $\mathrm{H}$ I and $\mathrm{H}_{2}$ content for the initial release of the GASS+COLD GASS sample, and found that the molecular-to-atomic gas mass ratio, 

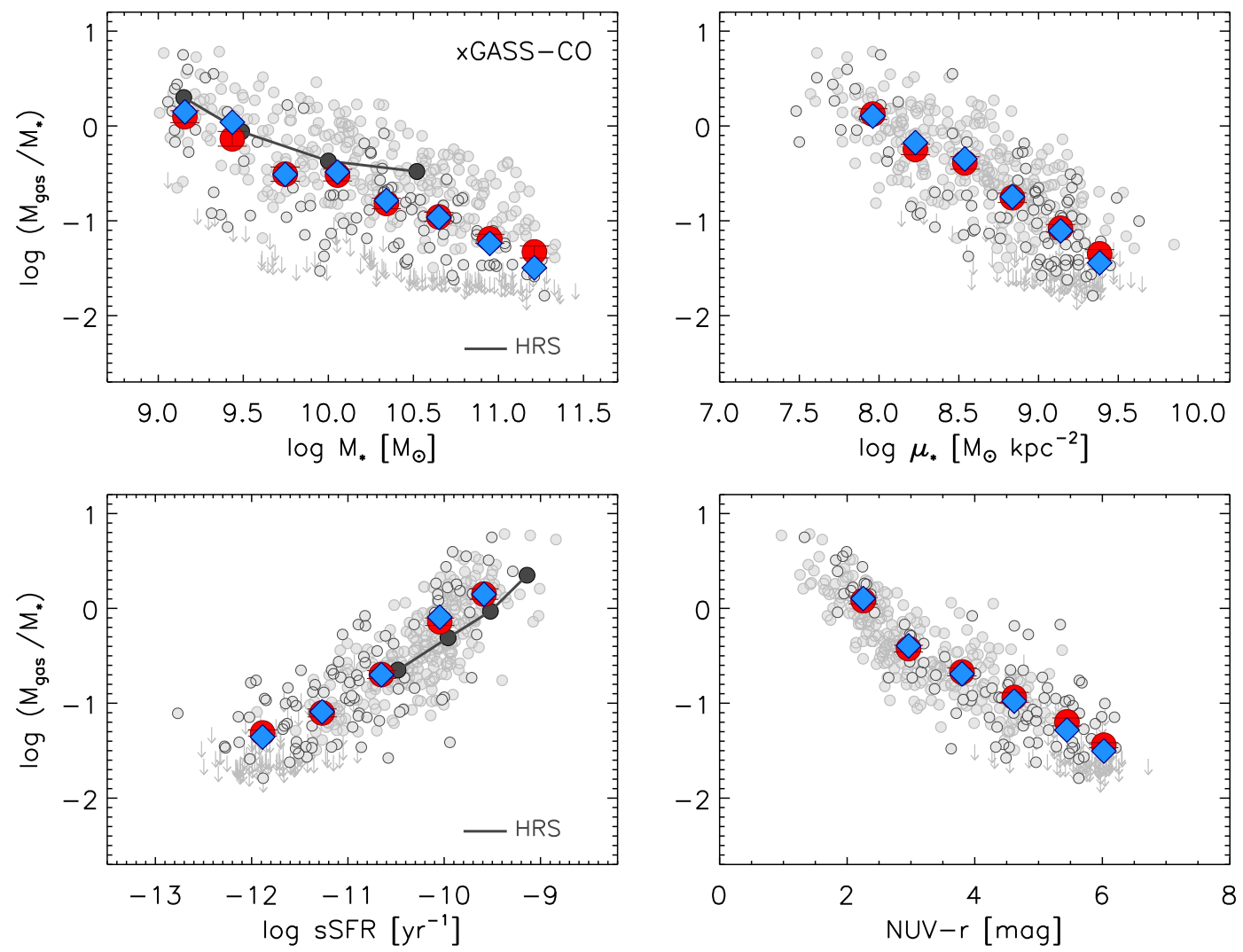

Figure 8. Total gas fraction scaling relations for the xGASS-CO sample. Small grey circles and downward arrows show individual detections and nondetections in both $\mathrm{H}_{\mathrm{I}}$ and $\mathrm{CO}$ lines; grey circles with darker contours indicate galaxies detected in $\mathrm{H}_{\mathrm{I}}$ or $\mathrm{CO}$, but not both. As in Fig. 6, large red circles and blue diamonds are weighted averages and weighted medians of the logarithms of the (total) gas fractions. These results are listed in Table 2. Black circles connected by lines in the left plots show the results from the Herschel Reference Survey (HRS; Boselli et al. 2014a).

$R_{\mathrm{mol}} \equiv M_{\mathrm{H}_{2}} / M_{\mathrm{HI}}$, weakly increases with stellar mass, stellar surface density and NUV $-r$ colour, but with over 0.4 dex of scatter (Saintonge et al. 2011). We also showed how $R_{\text {mol }}$ varies across the SFR $-M_{\star}$ plane for the full GASS+COLD GASS sample, and identified a region of unusually high values of $R_{\mathrm{mol}}$ $(>0.7)$ at high stellar masses and SFRs $\left(\log M_{\star}\left[\mathrm{M}_{\odot}\right]>10.8\right.$ and $-10.4<\log \mathrm{sSFR}\left[\mathrm{yr}^{-1}\right]<-9.6$; Saintonge et al. 2016). These galaxies are characterized by young stellar populations in their central regions (based on their $\mathrm{D}_{\mathrm{n}} 4000$ from SDSS fibre spectroscopy) and important bulge components $\left(\log \mu_{\star} \sim 8.9\right)$. Here, we extend these studies to lower stellar mass, focusing on the relation between galaxies within or outside the SFMS defined in the previous section. Galaxies with non-detections in both $\mathrm{H}_{\mathrm{I}}$ and $\mathrm{H}_{2}$ (magenta crosses in Fig. 7) are excluded from this analysis, because their $R_{\text {mol }}$ is unconstrained; we also exclude $\mathrm{H}$ I-confused detections (black-edged diamonds in Fig. 7), which leaves us with a sample of 328 galaxies.

Fig. 9 (top two panels) shows the molecular-to-atomic gas mass ratio as a function of stellar mass and stellar surface density for our sample; thick lines indicate running weighted medians of the logarithm of $R_{\text {mol }}$. The median $R_{\text {mol }}$ slowly increases with both stellar mass (from 9 per cent to 28 per cent) and stellar surface density (from 6 per cent to 25 per cent; see Table 3). A similar trend with stellar mass was also found by the APEX Low-redshift Legacy Survey for MOlecular Gas (ALLSMOG, Bothwell et al. 2014) and the HRS (Boselli et al. 2014a), which both combined COLD GASS data with new observations probing stellar masses below $10^{10} \mathrm{M}_{\odot}$. Furthermore, galaxies in the top two panels of Fig. 9 are colour-coded according to their distance from the SFMS (i.e. dist $_{\mathrm{MS}}=\log \mathrm{sSFR}-\log \mathrm{sSFR}_{\mathrm{MS}}$, see equation 2); negative values (redder colours) correspond to systems below the SFMS. Galaxies located below the SFMS typically have high stellar surface densities; this is better seen in the bottom panel, where $R_{\text {mol }}$ is plotted as a function of distance from the SFMS, and colour-coded by $\mu_{\star}$. On and above the SFMS (dashed line), bulge-dominated systems are displaced towards higher $R_{\mathrm{mol}}$ values.

The scatter in these plots is quite large, with $M_{\mathrm{H}_{2}} / M_{\mathrm{HI}}$ ratios that vary by almost two orders of magnitude across our sample. As seen in the middle panel, galaxies below the SFMS (which are typically bulge-dominated) seem to follow a different relation from starforming discs. This qualitatively agrees with the observation that HRS early-type galaxies detected in $\mathrm{H}$ i do not follow the same gas scaling relations as late-type ones (Boselli et al. 2014a). However, it is unclear if the observed scatter correlates more strongly with deviation from the SFMS or stellar surface density.

In order to gain further insight into what regulates the molecularto-atomic gas mass ratio of our sample, we compare $\mathrm{H}_{\mathrm{I}}$ and $\mathrm{H}_{2}$ gas fractions directly in Fig. 10. In the left-hand panel, galaxies on the SFMS are colour-coded by stellar surface density; the right-hand panel shows the complementary set of galaxies located outside the SFMS, with the same colour coding. Looking at the right-hand panel first, there is a general trend of increasing molecular gas fractions for increasing $M_{\mathrm{HI}} / M_{\star}$, with a clear dependence on stellar surface density. As can be seen, more bulge-dominated systems (redder colours in the figure) have systematically lower atomic and molecular gas fractions, while spanning the full range of $R_{\mathrm{mol}}$. Very interestingly, and contrary to the rest of the sample, the relation for 

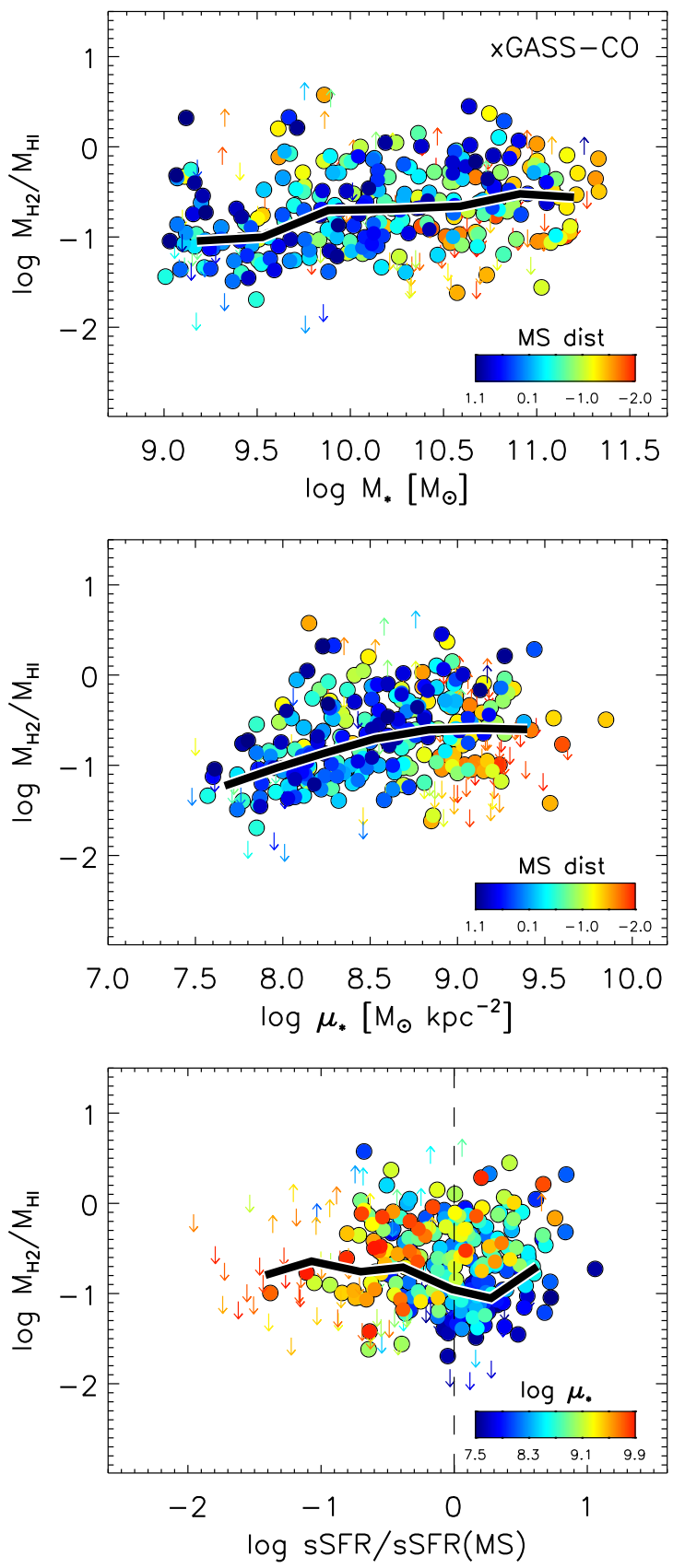

Figure 9. Molecular-to-atomic gas mass ratio as a function of stellar mass (top), stellar surface density (middle), and deviation from the star-forming main sequence (bottom). In each panel, circles are galaxies with both $\mathrm{H}_{\mathrm{I}}$ and $\mathrm{CO}$ detections, downward arrows are $\mathrm{H}$ I detections with $\mathrm{CO}$ upper limits, and upward arrows are $\mathrm{CO}$ detections with $\mathrm{H}$ I upper limits. Galaxies are colour-coded by distance from the SF sequence in the top two panels, and by $\mu_{\star}$ in the bottom one. Thick lines show running medians; only medians computed with at least 10 galaxies are shown.

SFMS galaxies (left) is nearly flat - selecting galaxies within $1 \sigma$ of the SFMS restricts $M_{\mathrm{H}_{2}} / M_{\star}$ to vary within a dex, whereas atomic gas fractions still span almost the entire range of the full sample. This is highlighted by the lines of constant molecular-to-atomic gas mass ratio, which increases from 3 per cent to 100 per cent from bottom to top, and shows that the observed variation of $\mathrm{R}_{\mathrm{mol}}$ is mostly driven by changes of the atomic gas reservoir - not the molecular one.
Table 2. Total gas fraction scaling relations for XGASS-CO.

\begin{tabular}{|c|c|c|c|c|}
\hline$x$ & $\langle x\rangle$ & $\left\langle M_{\mathrm{gas}} / M_{\star}\right\rangle^{a}$ & $\left(M_{\mathrm{gas}} / M_{\star}\right)^{b}$ & $N^{c}$ \\
\hline \multirow[t]{8}{*}{$\log M_{\star}$} & 9.16 & $0.098 \pm 0.064$ & 0.148 & 41 \\
\hline & 9.44 & $-0.136 \pm 0.077$ & 0.040 & 43 \\
\hline & 9.75 & $-0.509 \pm 0.076$ & -0.511 & 54 \\
\hline & 10.05 & $-0.518 \pm 0.062$ & -0.485 & 69 \\
\hline & 10.34 & $-0.817 \pm 0.055$ & -0.785 & 75 \\
\hline & 10.65 & $-0.958 \pm 0.048$ & -0.965 & 89 \\
\hline & 10.95 & $-1.190 \pm 0.048$ & -1.238 & 74 \\
\hline & 11.21 & $-1.328 \pm 0.064$ & -1.496 & 31 \\
\hline \multirow[t]{6}{*}{$\log \mu_{\star}$} & 7.96 & $0.125 \pm 0.057$ & 0.104 & 44 \\
\hline & 8.23 & $-0.247 \pm 0.056$ & -0.176 & 65 \\
\hline & 8.54 & $-0.390 \pm 0.067$ & -0.348 & 64 \\
\hline & 8.84 & $-0.757 \pm 0.049$ & -0.746 & 110 \\
\hline & 9.14 & $-1.079 \pm 0.040$ & -1.106 & 130 \\
\hline & 9.38 & $-1.348 \pm 0.045$ & -1.443 & 46 \\
\hline \multirow[t]{5}{*}{$\log \mathrm{sSFR}$} & -11.89 & $-1.313 \pm 0.032$ & -1.356 & 87 \\
\hline & -11.27 & $-1.102 \pm 0.042$ & -1.089 & 91 \\
\hline & -10.65 & $-0.698 \pm 0.042$ & -0.699 & 88 \\
\hline & -10.05 & $-0.141 \pm 0.039$ & -0.092 & 149 \\
\hline & -9.59 & $0.151 \pm 0.056$ & 0.150 & 44 \\
\hline \multirow[t]{6}{*}{$\mathrm{NUV}-r$} & 2.25 & $0.082 \pm 0.034$ & 0.104 & 95 \\
\hline & 2.96 & $-0.424 \pm 0.035$ & -0.394 & 80 \\
\hline & 3.80 & $-0.670 \pm 0.042$ & -0.689 & 69 \\
\hline & 4.62 & $-0.938 \pm 0.052$ & -0.978 & 76 \\
\hline & 5.44 & $-1.197 \pm 0.042$ & -1.282 & 82 \\
\hline & 6.02 & $-1.442 \pm 0.026$ & -1.504 & 52 \\
\hline
\end{tabular}

Notes. ${ }^{a}$ Weighted average of logarithm of gas fraction; H I mass of nondetections set to upper limit.

${ }^{b}$ Weighted median of logarithm of gas fraction; $\mathrm{HI}$ mass of non-detections set to upper limit.

${ }^{c}$ Number of galaxies in the bin.

Table 3. Molecular-to-atomic gas mass ratio scaling relations for xGASSCO.

\begin{tabular}{lcccc}
\hline$x$ & $\langle x\rangle$ & $\left\langle M_{\mathrm{H}_{2}} / M_{\mathrm{HI}}\right\rangle^{a}$ & $\left(M_{\mathrm{H}_{2}} / M_{\mathrm{HI}}\right)^{b}$ & $N^{c}$ \\
\hline $\log M_{\star}$ & 9.18 & $-0.903 \pm 0.070$ & -1.044 & 44 \\
& 9.53 & $-0.865 \pm 0.079$ & -1.003 & 38 \\
& 9.88 & $-0.660 \pm 0.073$ & -0.704 & 53 \\
& 10.22 & $-0.704 \pm 0.059$ & -0.691 & 59 \\
& 10.59 & $-0.662 \pm 0.057$ & -0.659 & 65 \\
& 10.91 & $-0.530 \pm 0.058$ & -0.521 & 50 \\
& 11.20 & $-0.578 \pm 0.075$ & -0.559 & 18 \\
$\log \mu_{\star}$ & 7.67 & $-1.132 \pm 0.066$ & -1.228 & 14 \\
& 7.96 & $-1.018 \pm 0.069$ & -1.032 & 41 \\
& 8.23 & $-0.682 \pm 0.066$ & -0.870 & 57 \\
& 8.53 & $-0.683 \pm 0.072$ & -0.706 & 49 \\
& 8.84 & $-0.583 \pm 0.060$ & -0.606 & 79 \\
& 9.13 & $-0.603 \pm 0.054$ & -0.591 & 70 \\
& 9.40 & $-0.602 \pm 0.122$ & -0.603 & 14
\end{tabular}

Notes. ${ }^{a}$ Weighted average of logarithm of $R_{\mathrm{mol}}$; $\mathrm{H}_{\mathrm{I}}$ and $\mathrm{H}_{2}$ masses of nondetections set to upper limits.

${ }^{b}$ Weighted median of logarithm of $R_{\mathrm{mol}} ; \mathrm{H}$ I and $\mathrm{H}_{2}$ masses of non-detections set to upper limits.

${ }^{c}$ Number of galaxies in the bin.

This finding suggests that the scatter in the total gas scaling relations might also be related to the variation of molecular-toatomic gas mass ratio, which is indeed the case. This is demonstrated in Fig. 11, which presents the relations of Fig. 8 with points colourcoded by $R_{\mathrm{mol}}$; grey arrows are galaxies with upper limits in both 

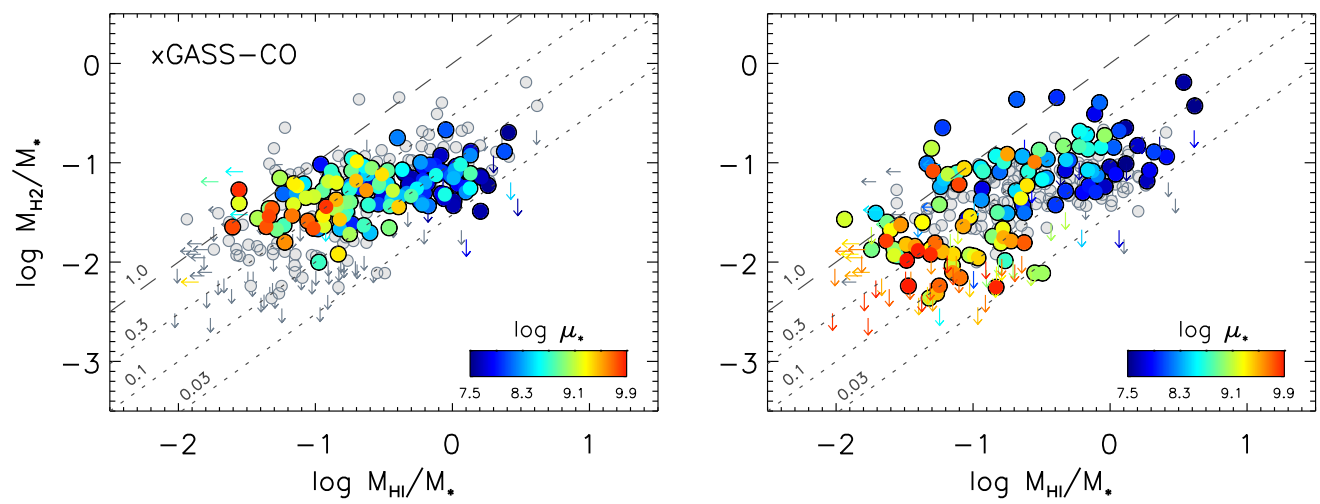

Figure 10. Comparison between $\mathrm{H}_{2}$ and $\mathrm{H}_{\mathrm{I}}$ gas fractions. Circles are galaxies detected in both $\mathrm{H}_{\mathrm{I}}$ and $\mathrm{H}_{2}$; downward and leftward arrows are $\mathrm{H}_{2}$ and $\mathrm{H}_{\mathrm{I}}$ upper limits, respectively. The two panels highlight galaxies within $1 \sigma$ from the SFMS (left; see Fig. 7) and outside the SFMS (right-hand panel), colour-coded according to their stellar surface density as indicated. Grey symbols in each panel show the excluded galaxies (i.e. correspond to the coloured points in the other panel). Also shown are lines of constant molecular-to-atomic gas mass ratio, with $M_{\mathrm{H}_{2}} / M_{\mathrm{HI}}$ values as labelled.
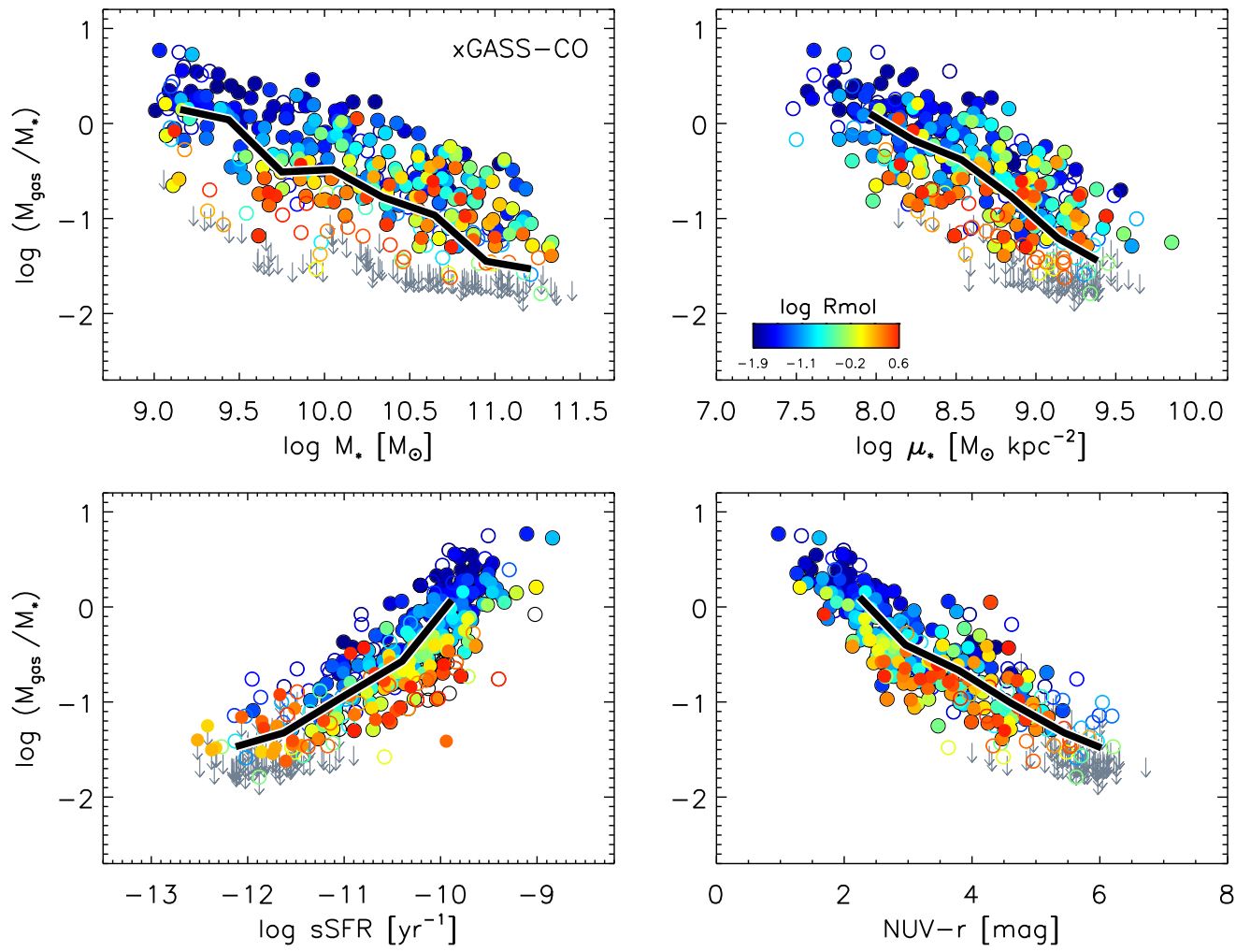

Figure 11. Total gas fraction scaling relations, colour-coded by molecular-to-atomic gas mass ratio. Empty circles indicate galaxies not detected in either $\mathrm{H}_{\mathrm{I}}$ or $\mathrm{H}_{2}$, and grey downward arrows are systems with upper limits in both gas phases, for which $R_{\mathrm{mol}}$ is not defined. This sample does not include $\mathrm{HI}$-confused galaxies, hence running medians (thick lines) are not identical to those in Fig. 8 (blue diamonds).

$\mathrm{H}_{\text {I }}$ and $\mathrm{H}_{2}$ for which $R_{\mathrm{mol}}$ is not defined. The variation of total gas fraction is clearly driven by a change in molecular-to-atomic gas mass ratio in all these plots. The secondary dependence on $R_{\mathrm{mol}}$ is most prominent at fixed specific SFR, where galaxies with smaller total gas reservoirs have larger values of $R_{\mathrm{mol}}$.

Lastly, the left-hand panel of Fig. 9 shows that, because $\mathrm{H}_{2}$ gas fractions are to first order roughly constant on the SFMS, the decrease of H I gas fractions leads to higher $R_{\text {mol }}$ for bulge-dominated systems, as observed in Fig. 9. It is tempting to interpret these trends with stellar surface density as suggestive of a causal link between galaxy structure and gas content. However, we obtain very similar results if we colour-code the galaxies in this figure by stellar mass (not shown), pointing out the difficulty of separating the effects of mass and structure using global measurements.

\section{COMPARISON WITH MODELS}

Gas fraction scaling relations for representative samples have a unique constraining power for galaxy formation models (e.g. Lagos et al. 2014, 2015; Popping, Somerville \& Trager 2014; Popping, Behroozi \& Peeples 2015; Bahé et al. 2016; Davé et al. 2017). Modern cosmological semi-analytic and hydrodynamical simulations successfully reproduce overall stellar and star formation properties of galaxies over cosmic time, but must rely on sub-resolution 
prescriptions to partition the cold gas into atomic and molecular phases and form stars. Comparisons between observed gas scaling relations from stellar mass-selected samples and simulated ones have highlighted areas where models need improvement (e.g. Kauffmann et al. 2012; Brown et al. 2017; Stevens \& Brown 2017; Zoldan et al. 2017).

In our companion paper (Saintonge et al. 2017), we compared $\mathrm{H}_{\mathrm{I}}$ and $\mathrm{H}_{2}$ gas fractions as a function of stellar mass with predictions from two large, state-of-the-art hydrodynamical simulations, MUFASA (Davé, Thompson \& Hopkins 2016; Davé et al. 2017), and the Evolution and Assembly of GaLaxies and their Environments (EAGLE, Schaye et al. 2015. We used their high resolution Recal-L025N0752 run).

Briefly, MUFASA directly tracks the amount of molecular gas formed in galaxies using a sub-resolution prescription (broadly following Krumholz et al. 2009). The atomic fraction is obtained by subtracting the molecular fraction from the neutral (self-shielded against the cosmic metagalactic flux) gas, and the global $\mathrm{H}$ i content of a galaxy is just the sum of the atomic gas that is bound to it.

For the EAGLE simulations, the partition of the ISM into its different phases was implemented by Lagos et al. (2015) in post-processing. The separation between ionized and neutral (selfshielded) gas is done according to the same prescription adopted by MUFASA (based on Rahmati et al. 2013); the neutral gas is then divided into $\mathrm{H}_{\mathrm{I}}$ and $\mathrm{H}_{2}$ phases following Gnedin \& Kravtsov (2011, hereafter GK11) or Krumholz (2013, hereafter K13). Both recipes give $\mathrm{H}_{2}$ fractions that depend on gas metallicity and strength of the interstellar radiation field, but the partition into $\mathrm{H}_{\mathrm{I}}$ and $\mathrm{H}_{2}$ phases relies on the assumption that the warm and cold components of the ISM are in pressure equilibrium (K13) or is based on metallicity, since $\mathrm{H}_{2}$ formation happens on dust grains (GK11).

The comparison with our results showed that both MUFASA and EAGLE simulations reproduce reasonably well the $\mathrm{H}$ I gas fractions in galaxies with $\log M_{\star}\left[\mathrm{M}_{\odot}\right]<10.5$, but significantly underpredict the amount of cold atomic gas in more massive galaxies. Contrary to the $\mathrm{H}_{\text {I }}$ phase, predictions of $\mathrm{H}_{2}$ gas fractions are very sensitive to the subgrid physics assumed to partition the ISM, and we found that none of these hydrodynamical simulations reproduce the molecular gas content of galaxies with $\log M_{\star}\left[\mathrm{M}_{\odot}\right]<10.5$ particularly well (Saintonge et al. 2017). The best agreement is with the EAGLE K13 prescription, whereas EAGLE GK11 and MUFASA produce galaxies with too much molecular gas.

Interestingly, despite the fact that $\mathrm{H}_{\mathrm{I}}$ and $\mathrm{H}_{2}$ gas fractions are not individually well reproduced by these hydrodynamical simulations across the full stellar mass range of our sample, Fig. 12 shows that the molecular-to-atomic gas mass ratio predicted by EAGLE is in overall better agreement with our observations (grey symbols, with the solid black line showing the median relation). In this figure, red and blue lines indicate median $R_{\text {mol }}$ values from MUFASA and Recal-L025N0752 EAGLE run, respectively; for the latter, light and dark blue lines correspond to the K13 and GK11 prescriptions. In order to be consistent with our observations, we applied our gas fraction limits to the simulated data sets, and excluded galaxies that would not be detected in both $\mathrm{H}$ I and $\mathrm{H}_{2}$ before computing the medians. MUFASA galaxies have molecular-to-atomic gas mass ratios $\sim 0.4$ dex higher than observed. This is because, to partly compensate for its lower resolution, MUFASA effectively employs a lowered density threshold for forming $\mathrm{H}_{2}$, which results in more ISM gas being molecular rather than atomic at a given stellar mass. The EAGLE K13 model provides an excellent match to our data, whereas the GK11 version slightly but systematically overestimates $R_{\text {mol }}$. We note that, while $\mathrm{H}_{\text {I }}$ gas fractions above $\log M_{\star}\left[\mathrm{M}_{\odot}\right] \sim 10.2$, are similarly underestimated in both subgrid

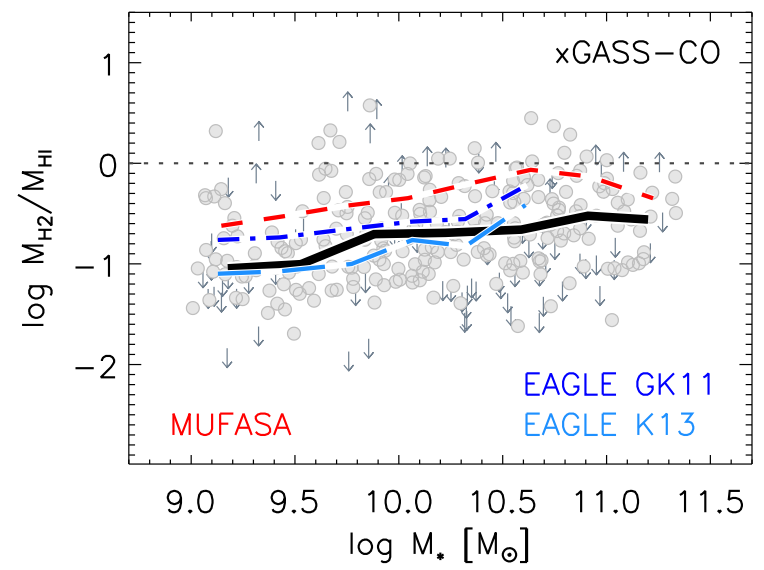

Figure 12. Comparison with hydrodynamical simulations. The molecularto-atomic gas mass ratio as a function of stellar mass is reproduced from the top panel of Fig. 9 (grey points), along with the median relation for our sample (black line). Predictions from MUFASA (Davé et al. 2016) and EAGLE (Lagos et al. 2015) hydrodynamical models are shown as red and blue lines, respectively. The two EAGLE models differ for the prescription adopted to partition the cold gas into atomic and molecular phases (GK11; K13). The horizontal dotted line is at $M_{\mathrm{H}_{2}} / M_{\mathrm{HI}}=1$, separating atomic- from molecular-dominated systems.

implementations, the $\mathrm{H}_{2}$ fractions are underestimated by $\mathrm{K} 13$ (thus getting $R_{\text {mol }}$ approximately correct) and overestimated for GK11, resulting in higher molecular-to-atomic gas mass ratios. This shows the importance of testing multiple gas scaling relations to constrain the physics implemented in simulations.

\section{SUMMARY AND CONCLUSIONS}

In this paper we presented $x$ GASS, the culmination of several years of effort to gather deep $\mathrm{H}_{\mathrm{I}}$ observations for a stellar mass-selected sample of $\sim 1200$ galaxies with homogeneously measured optical and SF properties. XGASS is the combination of the original GASS survey, which started in 2008 and targeted galaxies with stellar masses larger than $10^{10} \mathrm{M}_{\odot}$, and its extension to $M_{\star}=10^{9} \mathrm{M}_{\odot}$. Together, these surveys required $\sim 1300 \mathrm{~h}$ of Arecibo telescope time. Our unique approach of carrying out gas fraction-limited observations down to $M_{\mathrm{HI}} / M_{\star} \sim 2$ per cent allowed us to obtain stringent upper limits, which are essential to interpret variations of gas content as a function of galaxy properties.

We release here $\mathrm{H}$ I catalogues and spectra for the complete lowmass extension of GASS, which includes new Arecibo observations of 208 galaxies. By adding the correct proportion of ALFALFA $\mathrm{H}$ I-rich systems (not targeted by us to increase survey efficiency) to GASS and GASS-low data sets, we obtained a representative sample (in terms of H I content) of 1179 galaxies with stellar mass $10^{9} \leq M_{\star} / \mathrm{M}_{\odot} \leq 10^{11.5}$ in the local Universe $(0.01 \leq z \leq 0.05)$.

In addition to extending the $\mathrm{H}_{\mathrm{I}}$ scaling relations by one decade in stellar mass, we quantified total gas fraction scaling relations for the subset of 477 galaxies with molecular hydrogen mass estimates available, and explored molecular-to-atomic gas mass variations for galaxies detected in at least one of the two gas phases. $\mathrm{H}_{2}$ masses were obtained as part of the XCOLD GASS follow-up survey, which measured the $\mathrm{CO}(1-0)$ line emission of $\mathrm{xGASS}$ galaxies using the IRAM 30 m radio telescope (Saintonge et al. 2017). Our main results are summarized below.

(i) Atomic gas fractions increase from 2 per cent (set by the limit of our observations) to 81 percent with decreasing stellar mass, 
with no sign of a plateau. The tightest relation is with NUV $-r$ colour, which traces dust-unobscured star formation (as opposed to molecular gas fractions that correlate more strongly with sSFR, Saintonge et al. 2017).

(ii) On average, galaxies have gas reservoirs that remain dominated by atomic hydrogen across the full range of stellar masses probed by our survey (see also Fig. 12). Molecular-to-atomic hydrogen mass ratios weakly increase with stellar mass from 9 percent to 27 percent, but varying by two orders of magnitude across the sample.

(iii) Total gas fraction scaling relations closely resemble atomic ones, as expected from the fact that $\mathrm{H}_{\mathrm{I}}$ is the dominant gas phase. Below $\log M_{\star} / \mathrm{M}_{\odot} \sim 9.5$, the median galaxy has more mass in cold gas than stars. The scatter in the total gas fraction relations is driven by changes in $R_{\mathrm{mol}}$. At fixed specific SFR, galaxies with larger total gas reservoirs have smaller molecular-to-atomic gas mass ratios.

(iv) For galaxies on the star-forming sequence, variations of $R_{\mathrm{mol}}$ are mostly driven by changes of the $\mathrm{H}_{\mathrm{I}}$ reservoirs, with a clear dependence on stellar surface density. Bulge-dominated systems have $M_{\mathrm{H}_{2}} / M_{\mathrm{HI}}$ ratios that are typically three times larger than those of disc-dominated galaxies. This highlights once again the importance of galaxy structure, as traced by stellar surface density, in relation to the cold gas content of galaxies (see also Catinella et al. 2010; Saintonge et al. 2011, 2012; Brown et al. 2015).

When interpreting these results, one has to bear in mind that $\mathrm{H} \mathrm{I}$ and $\mathrm{H}_{2}$ line fluxes are measured with radio telescopes with vastly different beams $(\sim 3.5 \operatorname{arcmin}$ for Arecibo and $\sim 22 \operatorname{arcsec}$ for IRAM). We apply aperture corrections to recover global $\mathrm{H}_{2}$ masses, but none the less it is well known that most of the $\mathrm{HI}$ is distributed in the outer parts of galaxy discs, beyond the $\mathrm{H}_{2}$ dominated regions. Thus, our $M_{\mathrm{H}_{2}} / M_{\mathrm{HI}}$ ratios carry information on the global $\mathrm{H}_{\mathrm{I}}$ and $\mathrm{H}_{2}$ gas reservoirs available for future star formation, more than on the detailed conversion between the two. Even with this caveat, it remains very intriguing to investigate the reason(s) for the systematic variation of the molecular-to-atomic gas mass ratio with stellar surface density, and ultimately presence of a bulge component. While it remains difficult to establish if stellar mass or structure is more important in connection with the gas content of galaxies (at least using global quantities), there is no doubt that part of the scatter in all the relations presented in this work must be due to the fact that we normalize gas masses by stellar mass, which includes the bulge component, whereas the gas is found in the disc. We will address this issue in future work, by performing accurate photometric bulge-to-disc decompositions for xGASS galaxies to separate the total stellar mass into bulge and disc contributions, $M_{\star, \mathrm{B}}$ and $M_{\star, \mathrm{D}}$. We will then be able to investigate gas fraction scaling relations for the disc component alone (i.e. plotting $\left.M_{\mathrm{HI}} / M_{\star, \mathrm{D}}\right)$ and determine if and how these are affected by the presence of a bulge.

Statistical measurements of the cold gas content for stellar massselected samples are a crucial test-bed for models of galaxy formation. We presented an example by comparing molecular-to-atomic gas mass ratios measured from our sample with two state-of-theart hydrodynamical simulations, MUFASA and EAGLE, and noted how sometimes good agreement is obtained overall, even though the underlying distributions are not well reproduced. This is a complex parameter space, with several systematic trends that are still not completely understood, thus it is essential to test simulations with the largest possible combination of ISM components and galaxy properties - something that our large, homogeneous and very sensitive $\mathrm{xGASS}$ and $\mathrm{xCOLD}$ GASS surveys were precisely designed to provide.

\section{ACKNOWLEDGEMENTS}

We thank Claudia Lagos for making the results of her simulations available and for useful discussions, and an anonymous referee for a very careful reading of our paper and constructive comments. BC is the recipient of an Australian Research Council Future Fellowship (FT120100660). BC, SJ, and LC acknowledge support from the Australian Research Council's Discovery Projects funding scheme (DP150101734). APC acknowledges the support of STFC grant ST/P000541/1.

This research has made use of the NASA/IPAC Extragalactic Database (NED) which is operated by the Jet Propulsion Laboratory, California Institute of Technology, under contract with the National Aeronautics and Space Administration.

The Arecibo Observatory is operated by SRI International under a cooperative agreement with the National Science Foundation (AST-1100968), and in alliance with Ana G. Méndez-Universidad Metropolitana, and the Universities Space Research Association.

GALEX (Galaxy Evolution Explorer) is a NASA Small Explorer, launched in April 2003. We gratefully acknowledge NASA's support for construction, operation, and science analysis for the GALEX mission, developed in cooperation with the Centre National d'Etudes Spatiales (CNES) of France and the Korean Ministry of Science and Technology.

Funding for the SDSS and SDSS-II has been provided by the Alfred P. Sloan Foundation, the Participating Institutions, the National Science Foundation, the U.S. Department of Energy, the National Aeronautics and Space Administration, the Japanese Monbukagakusho, the Max Planck Society, and the Higher Education Funding Council for England. The SDSS Web Site is http://www.sdss.org/.

The SDSS is managed by the Astrophysical Research Consortium for the Participating Institutions. The Participating Institutions are the American Museum of Natural History, Astrophysical Institute Potsdam, University of Basel, University of Cambridge, Case Western Reserve University, University of Chicago, Drexel University, Fermilab, the Institute for Advanced Study, the Japan Participation Group, Johns Hopkins University, the Joint Institute for Nuclear Astrophysics, the Kavli Institute for Particle Astrophysics and Cosmology, the Korean Scientist Group, the Chinese Academy of Sciences (LAMOST), Los Alamos National Laboratory, the Max-Planck-Institute for Astronomy (MPIA), the MaxPlanck-Institute for Astrophysics (MPA), New Mexico State University, Ohio State University, University of Pittsburgh, University of Portsmouth, Princeton University, the United States Naval Observatory, and the University of Washington.

\section{REFERENCES}

Abazajian K. N. et al., 2009, ApJS, 182, 543

Accurso G. et al., 2017, MNRAS, 470, 4750

Bahé Y. M. et al., 2016, MNRAS, 456, 1115

Baldry I. K. et al., 2012, MNRAS, 421, 621

Barnes D. G. et al., 2001, MNRAS, 322, 486

Bianchi L., Conti A., Shiao B., 2014, Adv. Space Res., 53, 900

Bigiel F., Leroy A., Walter F., Brinks E., de Blok W. J. G., Madore B., Thornley M. D., 2008, AJ, 136, 2846

Bigiel F., Leroy A., Seibert M., Walter F., Blitz L., Thilker D., Madore B., 2010, ApJ, 720, L31

Binggeli B., Sandage A., Tammann G. A., 1985, AJ, 90, 1681

Blitz L., Rosolowsky E., 2006, ApJ, 650, 933

Boselli A. et al., 2010, PASP, 122, 261

Boselli A., Cortese L., Boquien M., 2014, A\&A, 564, A65 
Boselli A., Cortese L., Boquien M., Boissier S., Catinella B., Lagos C., Saintonge A., 2014a, A\&A, 564, A66

Boselli A., Cortese L., Boquien M., Boissier S., Catinella B., Gavazzi G., Lagos C., Saintonge A., 2014b, A\&A, 564, A67

Bothwell M. S. et al., 2014, MNRAS, 445, 2599

Brinks E., 1990, in Thronson H. A., Jr, Shull J. M., eds, Astrophysics and Space Science Library, Vol. 161, The Interstellar Medium in Galaxies. Kluwer, Dordrecht, p. 39

Brown T., Catinella B., Cortese L., Kilborn V., Haynes M. P., Giovanelli R., 2015, MNRAS, 452, 2479

Brown T. et al., 2017, MNRAS, 466, 1275

Catinella B., Cortese L., 2015, MNRAS, 446, 3526

Catinella B., Haynes M. P., Giovanelli R., 2007, AJ, 134, 334

Catinella B. et al., 2010, MNRAS, 403, 683

Catinella B. et al., 2012a, MNRAS, 420, 1959

Catinella B. et al., 2012b, A\&A, 544, A65

Catinella B. et al., 2013, MNRAS, 436, 34

Chabrier G., 2003, PASP, 115, 763

Cicone C. et al., 2017, A\&A, 604, A53

Cortese L. et al., 2016, MNRAS, 459, 3574

Cox D. P., 2005, ARA\&A, 43, 337

Davé R., Thompson R., Hopkins P. F., 2016, MNRAS, 462, 3265

Davé R., Rafieferantsoa M. H., Thompson R. J., Hopkins P. F., 2017, MNRAS, 467, 115

Dreyer J. L. E., 1888, Mem. RAS, 49, 1

Dreyer J. L. E., 1895, Mem. RAS, 51, 185

Dreyer J. L. E., 1908, Mem. RAS, 59, 105

Fu J., Guo Q., Kauffmann G., Krumholz M. R., 2010, MNRAS, 409, 515

Fumagalli M., Krumholz M. R., Prochaska J. X., Gavazzi G., Boselli A., 2009, ApJ, 697, 1811

Giovanelli R. et al., 2005, AJ, 130, 2598

Glover S. C. O., Clark P. C., 2012, MNRAS, 421, 9

Gnedin N. Y., Kravtsov A. V., 2011, ApJ, 728, 88 (GK11)

Haynes M. P. et al., 2011, AJ, 142, 170

Huang S., Haynes M. P., Giovanelli R., Brinchmann J., 2012, ApJ, 756, 113

Janowiecki S., Catinella B., Cortese L., Saintonge A., Brown T., Wang J., 2017, MNRAS, 466, 4795

Kalberla P. M. W., Kerp J., 2009, ARA\&A, 47, 27

Kauffmann G. et al., 2012, MNRAS, 422, 997

Kennicutt R. C., Evans N. J., 2012, ARA\&A, 50, 531

Klessen R. S., Glover S. C. O., 2016, Star Formation in Galaxy Evolution: Connecting Numerical Models to Reality, Saas-Fee Advanced Course, Vol. 43. Springer-Verlag, Berlin, p. 85

Krumholz M. R., 2013, MNRAS, 436, 2747 (K13)

Krumholz M. R., McKee C. F., Tumlinson J., 2009, ApJ, 693, 216

Lagos C. d. P. et al., 2015, MNRAS, 452, 3815

Lagos C. D. P., Baugh C. M., Zwaan M. A., Lacey C. G., Gonzalez-Perez V., Power C., Swinbank A. M., van Kampen E., 2014, MNRAS, 440, 920

Leroy A. K., Walter F., Brinks E., Bigiel F., de Blok W. J. G., Madore B., Thornley M. D., 2008, AJ, 136, 2782

Lilly S. J., Carollo C. M., Pipino A., Renzini A., Peng Y., 2013, ApJ, 772, 119

Martig M., Bournaud F., Teyssier R., Dekel A., 2009, ApJ, 707, 250

Martin D. C. et al., 2005, ApJ, 619, L1

McKee C. F., Ostriker J. P., 1977, ApJ, 218, 148

Meyer M. J. et al., 2004, MNRAS, 350, 1195

Meyer M., Robotham A., Obreschkow D., Westmeier T., Duffy A., StaveleySmith L., 2017, PASA, 34, 52

Moffett A. J. et al., 2016, MNRAS, 457, 1308

Nilson P., 1973, Uppsala General Catalogue of Galaxies. Astronomiska Observatorium, Uppsala

Obreschkow D., Heywood I., Klöckner H.-R., Rawlings S., 2009, ApJ, 702, 1321

Popping G., Somerville R. S., Trager S. C., 2014, MNRAS, 442, 2398

Popping G., Behroozi P. S., Peeples M. S., 2015, MNRAS, 449, 477
Rahmati A., Schaye J., Pawlik A. H., Raičevic M., 2013, MNRAS, 431, 2261

Saintonge A., 2007, AJ, 133, 2087

Saintonge A. et al., 2011, MNRAS, 415, 32

Saintonge A. et al., 2012, ApJ, 758, 73

Saintonge A. et al., 2016, MNRAS, 462, 1749

Saintonge A. et al., 2017, ApJS, 233, 22

Schaye J. et al., 2015, MNRAS, 446, 521

Seibert M. et al., 2012, American Astronomical Society Meeting Abstracts, Vol. 219, p. 340.01

Serra P. et al., 2012, MNRAS, 422, 1835

Springob C. M., Haynes M. P., Giovanelli R., Kent B. R., 2005, ApJS, 160, 149 (S05)

Stevens A. R. H., Brown T., 2017, MNRAS, 471, 447

Wang J. et al., 2011, MNRAS, 412, 1081

Wolfire M. G., Hollenbach D., McKee C. F., Tielens A. G. G. M., Bakes E. L. O., 1995, ApJ, 443, 152

Wong O. I. et al., 2006, MNRAS, 371, 1855

Wright E. L. et al., 2010, AJ, 140, 1868

Wyder T. K. et al., 2007, ApJS, 173, 293

Yang X., Mo H. J., van den Bosch F. C., Pasquali A., Li C., Barden M., 2007, ApJ, 671, 153

Young J. S. et al., 1995, ApJS, 98, 219

Young L. M. et al., 2011, MNRAS, 414, 940

Zoldan A., De Lucia G., Xie L., Fontanot F., Hirschmann M., 2017, MNRAS, 465,2236

Zwicky F., Herzog E., Wild P., Karpowicz M., Kowal C. T., 1961, Catalogue of Galaxies and of Clusters of Galaxies, Vol. I. California Institute of Technology, Pasadena

\section{SUPPORTING INFORMATION}

Supplementary data are available at MNRAS online.

Please note: Oxford University Press is not responsible for the content or functionality of any supporting materials supplied by the authors. Any queries (other than missing material) should be directed to the corresponding author for the article.

\section{APPENDIX A: DATA RELEASE}

We present here SDSS postage stamp images, Arecibo $\mathrm{H}$ I-line spectra, and catalogues of optical, UV, and $\mathrm{H}_{\mathrm{I}}$ parameters for the 208 GASS-low galaxies. The content of the tables is described below; notes on individual objects (marked with an asterisk in the last column of Tables A2 and A3) are reported in Appendix B.

\section{SDSS and GALEX data}

Table A1 lists optical and UV quantities for the 208 GASS-low galaxies, ordered by increasing right ascension:

Cols. 1 and 2: GASS and SDSS identifiers. Galaxies with six digit GASS IDs are part of GASS-low.

Col. 3: UGC (Nilson 1973), NGC (Dreyer 1888), or IC (Dreyer 1895,1908 ) designation, or other name, typically from the Catalog of Galaxies and Clusters of Galaxies (CGCG, Zwicky et al. 1961), or the Virgo Cluster Catalog (VCC, Binggeli, Sandage \& Tammann 1985).

Col. 4: SDSS redshift, $z_{\text {SDSS }}$. The typical uncertainty of SDSS redshifts for this sample is 0.0002 .

Col. 5: base-10 logarithm of the stellar mass, $M_{\star}$, in solar units. Stellar masses are obtained from the SDSS DR7 MPA/JHU catalogue (see footnote 2 in Section 2.3) and assume a Chabrier (2003) 
Table A1. SDSS and UV parameters of GASS-low galaxies.

\begin{tabular}{|c|c|c|c|c|c|c|c|c|c|c|c|c|c|c|}
\hline $\begin{array}{l}\text { GASS } \\
\text { (1) }\end{array}$ & $\begin{array}{l}\text { SDSS ID } \\
\text { (2) }\end{array}$ & $\begin{array}{l}\text { Other name } \\
\text { (3) }\end{array}$ & $\begin{array}{c}z_{\text {SDSS }} \\
\text { (4) }\end{array}$ & $\begin{array}{c}\log M_{\star} \\
\left(\mathrm{M}_{\odot}\right) \\
(5)\end{array}$ & $\begin{array}{c}R_{50, z} \\
(\operatorname{arcsec}) \\
\quad(6)\end{array}$ & $\begin{array}{c}R_{50} \\
(\operatorname{arcsec}) \\
(7)\end{array}$ & $\begin{array}{c}R_{90} \\
(\operatorname{arcsec}) \\
(8)\end{array}$ & $\begin{array}{c}\log \mu_{\star} \\
\left(\mathrm{M}_{\odot} \mathrm{kpc}_{(9)}^{-2}\right)\end{array}$ & $\begin{array}{c}\operatorname{ext}_{r} \\
(\mathrm{mag}) \\
(10)\end{array}$ & $\begin{array}{c}r \\
(\mathrm{mag}) \\
(11)\end{array}$ & $\begin{array}{l}(b / a)_{r} \\
(12)\end{array}$ & $\begin{array}{l}\text { incl } \\
\text { (deg) } \\
(13)\end{array}$ & $\begin{array}{c}\text { NUV }-r \\
(\mathrm{mag}) \\
(14)\end{array}$ & $\begin{array}{c}\text { SFR } \\
\left(\mathrm{M}_{(} \mathrm{yr}^{\mathrm{yr}^{-1}}\right)\end{array}$ \\
\hline 124009 & J000619.61+141938.7 & - & 0.0182 & 9.66 & 3.52 & 3.82 & 11.17 & 8.63 & 0.31 & 15.47 & 0.394 & 70 & 5.27 & 0.078 \\
\hline 124006 & J001947.33+003526.8 & - & 0.0177 & 9.75 & 3.47 & 3.36 & 10.58 & 8.76 & 0.07 & 14.75 & 0.753 & 42 & 3.82 & 0.539 \\
\hline 124004 & J002534.40+005048.6 & - & 0.0178 & 9.31 & 5.35 & 6.11 & 11.54 & 7.93 & 0.06 & 15.32 & 0.917 & 24 & 2.21 & 0.326 \\
\hline 124002 & J004903.69+152907.9 & - & 0.0183 & 9.25 & 2.60 & 2.57 & 6.02 & 8.48 & 0.17 & 15.72 & 0.479 & 64 & 2.33 & 0.209 \\
\hline 101030 & J014803.60+125604.6 & - & 0.0175 & 9.10 & 7.06 & 8.85 & 17.38 & 7.50 & 0.18 & 15.99 & 0.659 & 50 & 2.90 & 0.061 \\
\hline 101000 & J014853.12+132526.2 & - & 0.0155 & 9.41 & 4.28 & 3.99 & 12.06 & 8.35 & 0.18 & 15.86 & 0.787 & 39 & 4.91 & 0.096 \\
\hline 101012 & J014902.52+125539.0 & - & 0.0174 & 9.83 & 3.77 & 4.01 & 9.92 & 8.78 & 0.22 & 15.06 & 0.642 & 52 & 5.17 & 0.027 \\
\hline 101024 & J014917.63+132759.9 & - & 0.0166 & 9.28 & 3.81 & 4.18 & 9.82 & 8.26 & 0.21 & 16.17 & 0.735 & 44 & 5.57 & 0.067 \\
\hline 101016 & J014918.93+130252.0 & - & 0.0178 & 9.72 & 2.53 & 2.74 & 6.50 & 8.99 & 0.24 & 15.53 & 0.845 & 33 & 5.51 & 0.007 \\
\hline 101019 & J014920.31+131754.5 & - & 0.0171 & 9.06 & 4.78 & 4.57 & 11.24 & 7.83 & 0.21 & 16.68 & 0.673 & 49 & 3.92 & 0.092 \\
\hline
\end{tabular}

Note. The full version of this table is available online.

Table A2. Hi Properties of GASS-low detections.

\begin{tabular}{|c|c|c|c|c|c|c|c|c|c|c|c|c|c|}
\hline $\begin{array}{l}\text { GASS } \\
\text { (1) }\end{array}$ & $\begin{array}{l}\text { SDSS ID } \\
\text { (2) }\end{array}$ & $\begin{array}{c}z_{\text {SDSS }} \\
(3)\end{array}$ & $\begin{array}{c}T_{\text {on }} \\
(\min ) \\
(4)\end{array}$ & $\begin{array}{c}\Delta v \\
\left(\mathrm{~km} \mathrm{~s}^{-1}\right) \\
(5)\end{array}$ & $\begin{array}{c}z \\
(6)\end{array}$ & $\begin{array}{c}W_{50} \\
\left(\mathrm{~km} \mathrm{~s}^{-1}\right) \\
(7)\end{array}$ & $\begin{array}{c}W_{50}^{c} \\
\left(\mathrm{~km} \mathrm{~s}^{-1}\right) \\
(8)\end{array}$ & $\begin{array}{c}F_{\mathrm{HI}} \\
\left(\mathrm{Jy} \mathrm{km} \mathrm{s}^{-1}\right) \\
(9)\end{array}$ & $\begin{array}{c}\mathrm{rms} \\
(\mathrm{mJy}) \\
(10)\end{array}$ & $\begin{array}{l}\mathrm{S} / \mathrm{N} \\
(11)\end{array}$ & $\begin{array}{c}\log M_{\mathrm{HI}} \\
\left(\mathrm{M}_{\odot}\right) \\
(12)\end{array}$ & $\begin{array}{c}\log M_{\mathrm{HI}} / M_{\star} \\
\quad(13)\end{array}$ & $\begin{array}{c}Q \\
(14)\end{array}$ \\
\hline 124004 & J002534.40+005048.6 & 0.0178 & 5 & 12 & 0.017816 & $130 \pm 2$ & 122 & $0.71 \pm 0.07$ & 0.72 & 17.5 & 8.99 & -0.32 & 1 \\
\hline 124002 & J004903.69+152907.9 & 0.0183 & 4 & 12 & 0.018269 & $205 \pm 3$ & 195 & $0.67 \pm 0.11$ & 0.90 & 10.6 & 8.98 & -0.26 & $1^{*}$ \\
\hline 101021 & J011653.58+000911.2 & 0.0190 & 5 & 12 & 0.018956 & $180 \pm 4$ & 170 & $0.93 \pm 0.10$ & 0.88 & 15.9 & 9.15 & -0.06 & $1 *$ \\
\hline 101031 & $\mathrm{~J} 014755.16+124131.0$ & 0.0175 & 5 & 12 & 0.017465 & $173 \pm 3$ & 164 & $0.86 \pm 0.07$ & 0.67 & 19.7 & 9.05 & -0.12 & 1 \\
\hline 101016 & J014918.93+130252.0 & 0.0178 & 50 & 15 & 0.017242 & $207 \pm 48$ & 196 & $0.40 \pm 0.03$ & 0.20 & 24.5 & 8.70 & -1.01 & $5^{*}$ \\
\hline 101001 & $\mathrm{~J} 015032.27+133942.2$ & 0.0174 & 10 & 12 & 0.017372 & $202 \pm 5$ & 193 & $1.10 \pm 0.07$ & 0.56 & 27.9 & 9.15 & 0.07 & 1 \\
\hline 101018 & J015036.88+130636.8 & 0.0165 & 59 & 12 & 0.016531 & $69 \pm 7$ & 62 & $0.07 \pm 0.02$ & 0.24 & 7.5 & 7.94 & -1.31 & $1 *$ \\
\hline 107019 & $\mathrm{~J} 074842.59+263223.2$ & 0.0155 & 25 & 12 & 0.015537 & $172 \pm 3$ & 164 & $0.22 \pm 0.04$ & 0.33 & 10.1 & 8.35 & -0.81 & $1^{*}$ \\
\hline 107026 & J075433.01+294238.8 & 0.0167 & 5 & 10 & 0.016672 & $223 \pm 3$ & 215 & $3.58 \pm 0.11$ & 1.02 & 53.3 & 9.63 & 0.26 & 1 \\
\hline 108143 & J080038.05+133923.0 & 0.0158 & 23 & 12 & 0.015754 & $101 \pm 7$ & 94 & $0.26 \pm 0.04$ & 0.51 & 10.3 & 8.45 & -0.68 & $1^{*}$ \\
\hline
\end{tabular}

Note. The full version of this table is available online.

initial mass function. Over our stellar mass range, these values are believed to be accurate to better than 30 per cent.

Col. 6: radius containing 50 per cent of the Petrosian flux in $z$ band, $R_{50, z}$, in arcsec.

Cols. 7 and 8: radii containing 50 percent and 90 percent of the Petrosian flux in $r$ band, $R_{50}$, and $R_{90}$, respectively, in arcsec.

Col. 9: base-10 logarithm of the stellar mass surface density, $\mu_{\star}$, in $\mathrm{M}_{\odot} \mathrm{kpc}^{-2}$. This quantity is defined as $\mu_{\star}=M_{\star} /\left(2 \pi R_{50, z}^{2}\right)$, with $R_{50, z}$ in kpc units (computed using angular distances).

Col. 10: Galactic extinction in $r$ band, ext $_{r}$, in magnitudes, from SDSS.

Col. 11: $r$-band model magnitude from SDSS, $r$, corrected for Galactic extinction.

Col. 12: minor-to-major axial ratio from the exponential fit in $r$ band, $(b / a)_{r}$, from SDSS.

Col. 13: inclination to the line of sight, in degrees (see Catinella et al. $2012 \mathrm{~b}$ for details).
Col. 14: NUV $-r$ observed colour, corrected for Galactic extinction, in magnitudes (see Janowiecki et al. 2017).

Col. 15: SFR from NUV and WISE photometry, in $\mathrm{M}_{\odot} \mathrm{yr}^{-1}$ (see Janowiecki et al. 2017).

\section{H I source catalogues}

This data release includes 120 detections and 88 non-detections, for which we provide upper limits below. The measured $\mathrm{H}$ I parameters for the detected galaxies are listed in Table A2, ordered by increasing right ascension:

Cols. 1 and 2: GASS and SDSS identifiers. Galaxies with six digit GASS IDs are part of GASS-low.

Col. 3: SDSS redshift, $z$ sDSs.

Col. 4: on-source integration time of the Arecibo observation, $T_{\text {on }}$, in minutes. This number refers to on scans that were actually 
Table A3. GASS-low non-detections.

\begin{tabular}{|c|c|c|c|c|c|c|c|}
\hline $\begin{array}{l}\text { GASS } \\
\text { (1) }\end{array}$ & $\begin{array}{l}\text { SDSS ID } \\
\text { (2) }\end{array}$ & $\begin{array}{l}z_{\text {SDSS }} \\
(3)\end{array}$ & $\begin{array}{c}T_{\mathrm{on}} \\
(\mathrm{min}) \\
(4)\end{array}$ & $\begin{array}{c}\mathrm{rms} \\
(\mathrm{mJy}) \\
(5)\end{array}$ & $\begin{array}{c}\log M_{\mathrm{HI}, \lim } \\
\left(\mathrm{M}_{\odot}\right) \\
(6)\end{array}$ & $\begin{array}{c}\log M_{\mathrm{HI}, \lim } / M_{\star} \\
\text { (7) }\end{array}$ & $\begin{array}{c}\text { Note } \\
(8)\end{array}$ \\
\hline 124009 & J000619.61+141938.7 & 0.0182 & 90 & 0.16 & 7.95 & -1.70 & - \\
\hline 124006 & J001947.33+003526.8 & 0.0177 & 65 & 0.21 & 8.05 & -1.71 & - \\
\hline 101012 & $\mathrm{~J} 014902.52+125539.0$ & 0.0174 & 35 & 0.28 & 8.14 & -1.68 & $*$ \\
\hline 101024 & J014917.63+132759.9 & 0.0166 & 45 & 0.23 & 8.03 & -1.25 & - \\
\hline 101019 & J014920.31+131754.5 & 0.0171 & 68 & 0.19 & 7.97 & -1.09 & - \\
\hline 101011 & $\mathrm{~J} 014933.52+131400.0$ & 0.0167 & 20 & 0.39 & 8.26 & -1.69 & - \\
\hline 101017 & J014953.83+125833.7 & 0.0156 & 48 & 0.22 & 7.95 & -1.40 & - \\
\hline 101008 & J014954.10+130735.9 & 0.0170 & 48 & 0.21 & 8.02 & -1.33 & $*$ \\
\hline 101010 & J015015.67+130826.8 & 0.0161 & 39 & 0.26 & 8.04 & -1.13 & - \\
\hline 108111 & J080116.61+091553.3 & 0.0155 & 10 & 0.43 & 8.24 & -1.72 & - \\
\hline 108080 & J080134.48+091817.4 & 0.0153 & 36 & 0.30 & 8.07 & -1.25 & $*$ \\
\hline 108065 & J080158.49+150331.5 & 0.0161 & 5 & 0.65 & 8.45 & -1.65 & - \\
\hline 108022 & J080210.94+092141.8 & 0.0160 & 8 & 0.63 & 8.43 & -1.61 & $*$ \\
\hline 108052 & J080212.07+103234.1 & 0.0145 & 30 & 0.27 & 7.97 & -1.63 & - \\
\hline 108013 & J080237.85+093000.3 & 0.0159 & 10 & 0.59 & 8.40 & -1.69 & $*$ \\
\hline
\end{tabular}

Note. The full version of this table is available online.

combined, and does not account for possible losses due to RFI excision (usually negligible).

Col. 5: velocity resolution of the final, smoothed spectrum in $\mathrm{km} \mathrm{s}^{-1}$. In general, lower signal-to-noise detections require more smoothing in order to better identify the edges and peaks of the Hi profiles, needed to measure the Hi parameters.

Col. 6: redshift, $z$, measured from the $\mathrm{H}_{\mathrm{I}}$ spectrum. The error on the corresponding heliocentric velocity, $c z$, is half the error on the width, tabulated in the following column.

Col. 7: observed velocity width of the source line profile in $\mathrm{km}$ $\mathrm{s}^{-1}, W_{50}$, measured at the 50 percent level of each peak. Briefly, we fit straight lines to the sides of the $\mathrm{H}_{\text {I }}$ profile, and identify the velocities $c z_{r}, c z_{a}$ corresponding to the 50 per cent peak flux density (from the fits) on the receding and approaching sides, respectively (see section 2.2 of Catinella, Haynes \& Giovanelli 2007, for more details). The observed width is just the difference between these two velocities (and the H I redshift is given by their average). The error on the width is the sum in quadrature of the statistical and systematic uncertainties in $\mathrm{km} \mathrm{s}^{-1}$. Statistical errors depend primarily on the signal to noise of the $\mathrm{H}$ i spectrum, and are obtained from the rms noise of the linear fits to the edges of the Hi profile. Systematic errors depend on the subjective choice of the H I signal boundaries (see Catinella et al. 2010), and are negligible for most of the galaxies in our sample (see also Appendix B).

Col. 8: velocity width corrected for instrumental broadening and cosmological redshift only, $W_{50}^{c}$, in $\mathrm{km} \mathrm{s}^{-1}$ (see Catinella et al. $2012 \mathrm{~b}$ for details). No inclination or turbulent motion corrections are applied.

Col. 9: integrated H I-line flux density in $\mathrm{Jy} \mathrm{km} \mathrm{s}^{-1}, F_{\mathrm{HI}} \equiv \int S \mathrm{~d} v$, measured on the smoothed and baseline-subtracted spectrum (observed velocity frame). The reported uncertainty is the sum in quadrature of the statistical and systematic errors (see col. 7). The statistical errors are calculated according to equation (2) of S05 (which includes the contribution from uncertainties in the baseline fit).

Col. 10: rms noise of the observation in mJy, measured on the signaland RFI-free portion of the smoothed spectrum.

Col. 11: signal-to-noise ratio of the H i spectrum, S/N, estimated following Saintonge (2007) and adapted to the velocity resolution of the spectrum. This is the definition of S/N adopted by ALFALFA, which accounts for the fact that for the same peak flux a broader spectrum has more signal.

Col. 12: base-10 logarithm of the $\mathrm{H}_{\mathrm{I}}$ mass, $M_{\mathrm{HI}}$, in solar units, computed via:

$\frac{M_{\mathrm{HI}}}{\mathrm{M}_{\odot}}=\frac{2.356 \times 10^{5}}{(1+z)^{2}}\left[\frac{d_{\mathrm{L}}(z)}{\mathrm{Mpc}}\right]^{2}\left(\frac{\int S \mathrm{~d} v}{\mathrm{Jy} \mathrm{km} \mathrm{s}^{-1}}\right)$,

where $d_{\mathrm{L}}(z)$ is the luminosity distance to the galaxy at redshift $z$ as measured from the $\mathrm{H}_{\mathrm{I}}$ spectrum in the observed velocity frame (Obreschkow et al. 2009; Meyer et al. 2017).

Col. 13: base-10 logarithm of the $\mathrm{H}_{\mathrm{I}}$ mass fraction, $M_{\mathrm{HI}} / M_{\star}$.

Col. 14: quality flag, $Q$ ( $1=$ good, $2=$ marginal, and $5=$ confused). An asterisk indicates the presence of a note for the source in Appendix B. Code 1 indicates reliable detections, with an $\mathrm{S} / \mathrm{N}$ ratio of order of 6.5 or higher. Marginal detections have lower $\mathrm{S} / \mathrm{N}$ (between 5 and 6.5), thus more uncertain $\mathrm{H}_{\mathrm{I}}$ parameters, but are still secure detections, with $\mathrm{H}$ I redshift consistent with the SDSS one. We flag galaxies as 'confused' when most of the H I emission is believed to originate from another source within the Arecibo beam. For some of the galaxies, the presence of small companions within the beam might contaminate (but is unlikely to dominate) the $\mathrm{H}$ signal - this is just noted in Appendix B.

Table A3 gives the derived $\mathrm{H}$ I upper limits for the non-detections. Columns $1-4$ and 5 are the same as columns $1-4$ and 10 in Table A2, respectively. Column 6 lists the upper limit on the H i mass in solar units, $\log M_{\mathrm{HI}, \mathrm{lim}}$, computed assuming a $5 \sigma$ signal with 

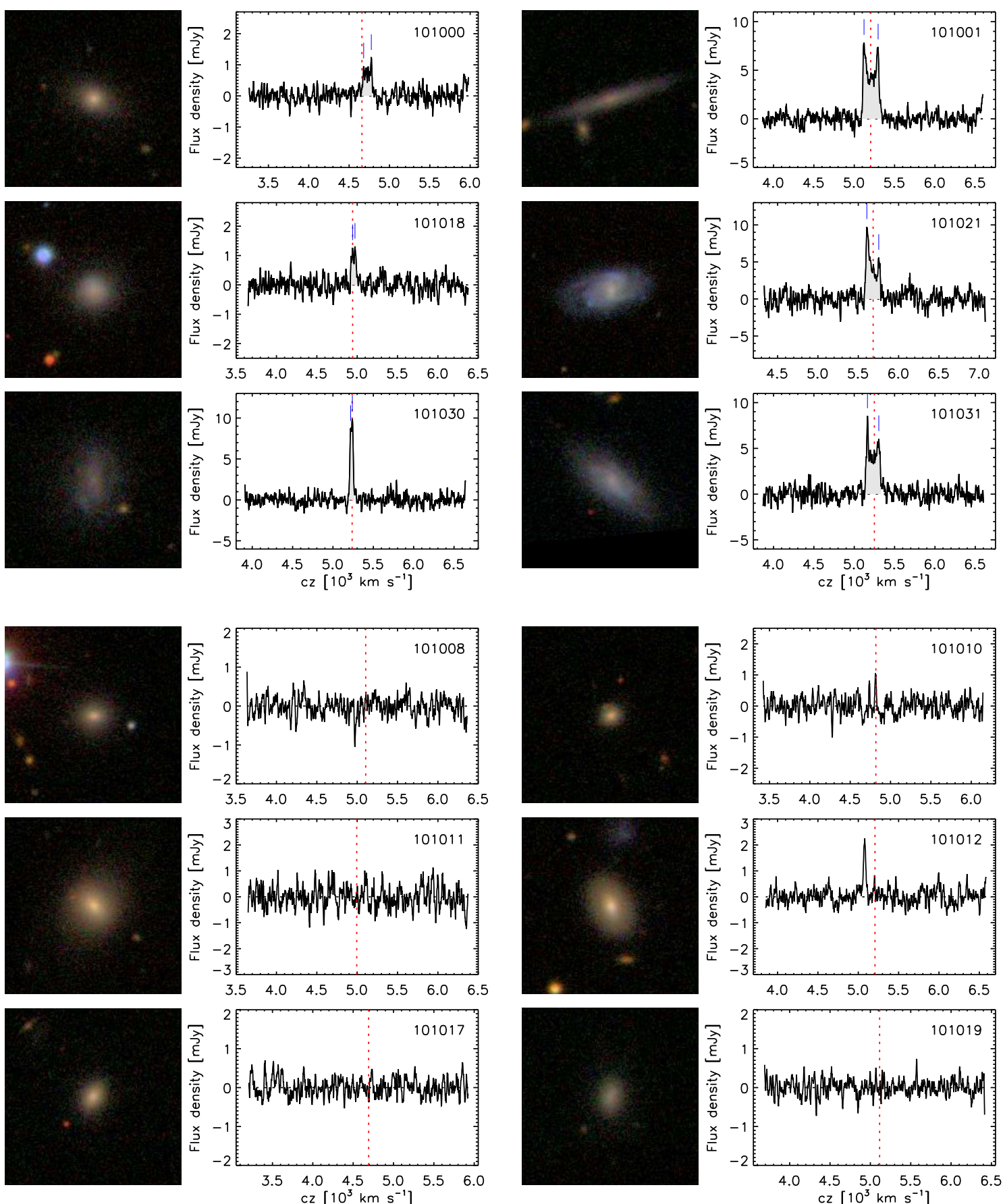

Figure A1. SDSS postage stamp images ( 1 arcmin square) and $\mathrm{H}$ I-line profiles of GASS-low galaxies, ordered by increasing GASS number (indicated in each spectrum). Top three rows: H i detections. The H i spectra are calibrated, smoothed, and baseline subtracted. A dotted line and two dashes indicate the heliocentric velocity corresponding to the SDSS redshift and the two peaks used for width measurement, respectively. Bottom three rows: non-detections. This is a sample of the complete figure, which is available in the online version of the article.

$200 \mathrm{~km} \mathrm{~s}^{-1}$ velocity width, if the spectrum was smoothed to $100 \mathrm{~km}$ $\mathrm{s}^{-1}$. Column 7 gives the corresponding upper limit on the gas fraction, $\log M_{\mathrm{HI}, \lim } / M_{\star}$. An asterisk in column 8 indicates the presence of a note for the galaxy in Appendix B.

\section{SDSS postage stamps and $\mathrm{H}$ I spectra}

Fig. A1 shows SDSS images and Arecibo H I spectra for a subset of galaxies included in this data release (top three rows: H I de- tections; bottom three rows: non-detections). The objects in each figure (detections and non-detections) are ordered by increasing GASS number, indicated on the top right corner of each spectrum. The SDSS images show a 1 arcmin square field, i.e. only the central part of the region sampled by the Arecibo beam (the half power full width of the beam is $\sim 3.5$ arcmin at the frequencies of our observations). Therefore, companions that might be detected in our spectra typically are not visible in the postage stamps, but they are noted in Appendix B. The H I spectra are always displayed over a 
$3000 \mathrm{~km} \mathrm{~s}^{-1}$ velocity interval, which includes the full $12.5 \mathrm{MHz}$ bandwidth adopted for our observations. The $\mathrm{HI}$-line profiles are calibrated, smoothed (to a velocity resolution between 5 and $15 \mathrm{~km}$ $\mathrm{s}^{-1}$ for the detections, as listed in Table A2, or to $15 \mathrm{~km} \mathrm{~s}^{-1}$ for the non-detections), and baseline subtracted. A red, dotted line indicates the heliocentric velocity corresponding to the optical redshift from SDSS. For the Hi detections, the shaded area and two vertical dashes show the part of the profile that was integrated to measure the $\mathrm{H}_{\mathrm{I}}$ flux and the peaks used for width measurement, respectively.

\section{APPENDIX B: NOTES ON INDIVIDUAL OBJECTS}

We list here notes for galaxies marked with an asterisk in the last column of Tables A2 and A3. The galaxies are ordered by increasing GASS number. In what follows, AA2 is the abbreviation for ALFALFA detection code 2.

\section{Detections (Table A2)}

101016 - asymmetric profile, uncertain width; confused with NGC $675\left(c z=5335 \mathrm{~km} \mathrm{~s}^{-1}\right.$ from NED) $\sim 2.5 \operatorname{arcmin~W}$; also notice large elliptical $\sim 1$ arcmin W (NGC 677, $5082 \mathrm{~km} \mathrm{~s}^{-1}$ ). Several small blue galaxies around, $z>0.08$.

101018 - polarization mismatch.

101021 - RFI spike at $1392 \mathrm{MHz}\left(\sim 6120 \mathrm{~km} \mathrm{~s}^{-1}\right)$; early-type galaxy $\sim 1$ arcmin SE has $z=0.048$

101030 - several galaxies within 3 arcmin, $z>0.06$.

107019 - small blue galaxy $\sim 1$ arcmin $\mathrm{W}, z=0.105$.

108011 - blue galaxy $\sim 1.5 \operatorname{arcmin} \mathrm{SW}, z=0.052$.

108014 - blend with large, edge-on disc $\sim 2$ arcmin SE (SDSS $\mathrm{J} 081011.20+245334.7, z=0.014056$ ); the face-on spiral $\sim 3$ arcmin $\mathrm{S}$ haz $z=0.081$.

108019 - blend with blue companion $\sim 2$ arcmin W (SDSS $\mathrm{J} 081002.90+224623.1, z=0.015736, c z=4718 \mathrm{~km} \mathrm{~s}^{-1}$ ).

108024 - confused/blend with two large spirals $\sim 1$ arcmin NW (SDSS J082401.55+210138.3, $z=0.015483$ ) and $\sim 2.5 \operatorname{arcmin~SW~}$ (SDSS J082355.28+205831.6, $z=0.015708$ ).

108029 - blend; interacting with large, blue companion $1.8 \operatorname{arcmin} \mathrm{N}$ (UGC 4264, $z=0.013642$ ).

108049 - AA2

108051 - AA2.

108078 - small blue galaxy $\sim 1$ arcmin $\mathrm{W}$ has no redshift; blue galaxy $\sim 1.5 \operatorname{arcmin} \mathrm{S}$ has $z=0.07$.

108093 - blue spiral 2 arcmin W, SDSS J083520.21+233943.2, has $z=0.039$.

108097 - small blue galaxy $\sim 3 \operatorname{arcmin} \mathrm{W}, z=0.130$.

108129 - two small blue galaxies $\sim 2 \operatorname{arcmin} \mathrm{W}, z>0.06$.

108140 - a few small galaxies within 3.5 arcmin, all in the background or without optical redshift; AA2.

108143 - face-on companion $\sim 2.5$ arcmin $\mathrm{N}$ (SDSS $\mathrm{J} 080037.51+134150.4, z=0.01525, c z=4572 \mathrm{~km} \mathrm{~s}^{-1}$ ) not detected; blue galaxy $\sim 40 \operatorname{arcsec} \mathrm{W}$, SDSS J080035.43+133936.8, has $z=0.073$.

108145 - polarization mismatch; high-frequency edge uncertain, systematic error. Companion $\sim 2 \operatorname{arcmin} W$ (SDSS J080158.07+092324.2, $z=0.013337, c z=3998 \mathrm{~km} \mathrm{~s}^{-1}$ ) and two early types $\sim 1.5$ arcmin SE (SDSS J080210.94+092141.7, $c z=4795 \mathrm{~km} \mathrm{~s}^{-1}$ ) and $\sim 2 \operatorname{arcmin} \mathrm{E}$ (NGC 2511, $c z=4467 \mathrm{~km}$

$\mathrm{s}^{-1}$ from NED) not detected.

109009 - near bright star; AA2.

109020 - small blue galaxy $\sim 2 \operatorname{arcmin} \mathrm{NE}, z=0.13$; AA2.
109034 - high-frequency edge uncertain, systematic error.

109058 - galaxy $\sim 2.5$ arcmin SE has $z=0.132$.

109077 - AA2.

109079 - large, face-on blue companion $\sim 1.5$ arcmin NE (UGC 5344, $c z \sim 4135 \mathrm{~km} \mathrm{~s}^{-1}$ ) also detected.

109083 - AA2.

109094 - small blue galaxy $\sim 2.5 \operatorname{arcmin} \mathrm{W}, z=0.012$.

109108 - AA2

109120 - small galaxy $\sim 1.5 \operatorname{arcmin} \mathrm{W}, z=0.09$; AA2.

109126 - AA2.

109129 - blend with blue companion $\sim 1$ arcmin E (SDSS

J090133.86+123931.6, $z=0.019761$ ).

109135 - AA2.

110013 - AA2.

110019 - galaxy $\sim 1.5$ arcmin SW has $z=0.143$.

110038 - $264 \mathrm{mJy}$ continuum source at 2 arcmin, but very strong signal.

110054 - blue, edge-on companion $\sim 3.5$ arcmin SE (SDSS $\mathrm{J} 104410.01+221233.2, z=0.019602$ ), unlikely to contribute significantly to the signal; early-type galaxy $\sim 1 \operatorname{arcmin} \mathrm{W}$ has $z=0.110$.

110057 - blend with large, blue companion $\sim 2$ arcmin NW (SDSS J102916.83+260557.2, $z=0.01699$ ).

110080 - two blue discs $\sim 2.5 \operatorname{arcmin} \mathrm{NW}$ and $\sim 2.5 \operatorname{arcmin} \mathrm{N}$ have redshifts $z=0.046$ and $z=0.162$, respectively.

111004 - blend with spectacular blue companion $\sim 3 \operatorname{arcmin} \mathrm{E}$ (SDSS J115805.22+275243.8, $z=0.011149$ ).

111047 - small blue galaxy $\sim 0.5$ arcmin NE, no optical $z$.

110058 - blend with blue companion $\sim 3$ arcmin N (SDSS $\mathrm{J} 101823.53+131642.1, z=0.018273$ ).

111053 - edge-on galaxy $\sim 2 \operatorname{arcmin} \mathrm{NW}, z=0.067$.

111063 - polarization mismatch; large blue galaxy $\sim 4$ arcmin NW (NGC 4005, cz=4469 $\mathrm{km} \mathrm{s}^{-1}$ ) not detected; blue disc $\sim 4$ arcmin W (SDSS J115809.49+250520.0, $z=0.014286$, $4283 \mathrm{~km} \mathrm{~s}^{-1}$ ), significant contamination unlikely.

111086 - blend with blue companion $\sim 3 \operatorname{arcmin} \mathrm{N}$ (SDSS $\mathrm{J} 113913.53+150215.7, z=0.014011)$.

112035 - early-type companion $\sim 2 \operatorname{arcmin}$ SE, SDSS $\mathrm{J} 125910.30+273711.5\left(z=0.01916,5744 \mathrm{~km} \mathrm{~s}^{-1}\right)$; AA2.

112068 - high-frequency edge uncertain, systematic error. Large, red disc $\sim 1.5 \operatorname{arcmin} \mathrm{NW}$ (NGC 4063, $c z=4917 \mathrm{~km} \mathrm{~s}^{-1}$ ) not detected; disc $\sim 2.5$ arcmin $\mathrm{E}$ has $z=0.024$.

112080 - blend: most of the $\mathrm{H}_{\mathrm{I}}$ signal comes from large, blue companion $\sim 2$ arcmin $\mathrm{E}$ (NGC 4615, $z=0.015731$ from NED); also notice spiral galaxy $\sim 3 \operatorname{arcmin} S$ at the same redshift (SDSS J124131.46+260233.5, $z=0.015957)$

112014 - confused with face-on, blue companion $\sim 20 \operatorname{arcsec} W$ (SDSS J120429.88+022654.5, $z=0.020141,6038 \mathrm{~km} \mathrm{~s}^{-1}$ ); blue galaxy $\sim 2$ arcmin W (SDSS J120424.60+022732.8, $c z=6730 \mathrm{~km}$ $\mathrm{s}^{-1}$ ) not detected.

112112 - two small galaxies $\sim 1.5$ and $\sim 2$ arcmin NE, no $z$.

112116 - galaxies $\sim 1$ arcmin SW and 2 arcmin $S$ in the background $(z=0.021$ and $z=0.025$, respectively).

113010 - RFI spike at $1408 \mathrm{MHz}\left(\sim 2650 \mathrm{~km} \mathrm{~s}^{-1}\right)$.

113011 - disc galaxy $\sim 2.5 \operatorname{arcmin} \mathrm{NE}$ has $z=0.112$

113038 - tiny blue comp. $\sim 2.5$ arcmin SW, SDSS $\mathrm{J} 132408.93+355317.6(z=0.018561)$.

113091 - three small blue galaxies within $3 \operatorname{arcmin}, z>0.04$.

113115 - small blue galaxies $\sim 1.5 \operatorname{arcmin} \mathrm{N}$ and $\sim 3 \operatorname{arcmin} \mathrm{SW}$ in the background $(z>0.04)$.

113118 - two galaxies $\sim 2.5 \operatorname{arcmin} \mathrm{SW}$ and $\sim 3 \operatorname{arcmin} \mathrm{NW}$ have $z=0.06$. 
113122 - low-frequency edge uncertain, systematic error. Several galaxies within 3 arcmin, $z>0.05$.

113123 - blue disc galaxy $\sim 4$ arcmin NE, $z=0.024$.

113124 - small blue galaxy $\sim 2 \operatorname{arcmin} \mathrm{NE}, z=0.06$. Unclear what is causing the feature at $1398.5-99 \mathrm{MHz}\left(\sim 4600 \mathrm{~km} \mathrm{~s}^{-1}\right)$, but it is present in both polarizations.

114001 - small blue galaxy $\sim 45 \operatorname{arcsec} \mathrm{W}$, no optical $z$.

114005 - confused/blend with blue companion $\sim 2$ arcmin NW (IC 1013, $z=0.015341,4599 \mathrm{~km} \mathrm{~s}^{-1}$ ); three large galaxies within 3 arcmin at the same redshift $(z=0.015)$, and a smaller one 2 arcmin $\mathrm{E}(z=0.013)$.

114033 - blend with two large, blue companions $\sim 1$ arcmin NE (SDSS J141216.00+155247.4, $z=0.017549)$ and $\sim 2 \operatorname{arcmin}$ SE (NGC 5504, $z=0.017505$ from NED).

114044 - profile edges uncertain, systematic error. Small blue galaxy $\sim 45 \operatorname{arcsec} \mathrm{SW}$, no optical $z$.

114047 - blend/confused with blue triplet $\sim 2.5$ arcmin E (SDSS J140520.30+102438.6, $z=0.018738 ;$ SDSS $\mathrm{J} 140519.91+102445.6$, no $z ;$ SDSS J140520.30+102452.9, $z=0.018332$ ); also notice large early-type $\sim 2 \operatorname{arcmin} \mathrm{E}$ (SDSS $\mathrm{J} 140516.52+102551.7, z=0.017949)$.

114076 - small blue galaxy $\sim 1.5 \operatorname{arcmin} \mathrm{N}$, no $z$; AA2.

114077 - early-type galaxy $\sim 2 \operatorname{arcmin} \mathrm{E}, z=0.030$; edge-on disc $\sim 0.5 \operatorname{arcmin} \mathrm{W}$, no optical $z$.

114091 - large spiral $\sim 1.5$ arcmin NE (UGC 9165, $c z=5259 \mathrm{~km}$ $\mathrm{s}^{-1}$ from NED) also detected; face-on spiral $\sim 2.5$ arcmin $\mathrm{SW}$ has $z=0.079$. Absorption-like feature at $\sim 5050 \mathrm{~km} \mathrm{~s}^{-1}$ is not RFI and is present in both polarizations (nothing obvious in the off position, just a few small blue galaxies without redshifts).

114110 - blend/confused with large blue companion $\sim 20$ arcsec S, NGC $5491(z=0.019647$ from NED).

114115 - small blue galaxy $\sim 3.5$ arcmin NE, $z=0.025$.

$\mathbf{1 1 4 1 4 4}$ - 120 mJy continuum source at 2 arcmin, standing waves. AA2.

122001 - two large galaxies $\sim 2 \operatorname{arcmin} \mathrm{NE}$ and $3 \operatorname{arcmin} \mathrm{E}$ have $z=0.037 ;$ AA2.

122002 - strong signal detected on top of long standing wave (in polarization A).

124002 - AA2.

124012 - galaxy $\sim 1.5 \operatorname{arcmin} \mathrm{NE}, z=0.15$; AA2.

\section{Non-detections (Table A3)}

101008 - blue disc $\sim 2 \operatorname{arcmin} \mathrm{N}$ has $z=0.034$.

101012 - detected companion $\left(c z=5080 \mathrm{~km} \mathrm{~s}^{-1}\right)$, most likely the blue smudge $\sim 10 \operatorname{arcsec} N$ (SDSS J014902.23+125603.2, no optical redshift).

108013 - galaxy $\sim 3$ arcmin SW, SDSS J080229.78+092743.2 $\left(z=0.014515,4351 \mathrm{~km} \mathrm{~s}^{-1}\right)$, also not detected.

108022 - blue companion $\sim 1 \operatorname{arcmin} \mathrm{NW}$

(SDSS

J080206.54+092238.3, $z=0.013795)$, also not detected.

108080 - blue disc galaxy $\sim 2 \operatorname{arcmin} \mathrm{W}$ has $z=0.047$.

108101 - perhaps hint of $\mathrm{H}$ I galaxy signal.

108114 - blue companion $~ 3.5 \operatorname{arcmin} \mathrm{N}$

(SDSS

J081919.78+210331.8, $z=0.016114$ ), also not detected.

109022 - several galaxies within 3 arcmin, all in the background or without optical redshift.

109036 - AA2.

109064 - detected large spiral $\sim 2 \operatorname{arcmin}$ S, (NGC 2874, $\left.z=0.012573,3769 \mathrm{~km} \mathrm{~s}^{-1}\right)$; also notice large elliptical $\sim 2$ arcmin $\mathrm{SW}$, (NGC 2872, $3196 \mathrm{~km} \mathrm{~s}^{-1}$ from NED).
109075 - low surface brightness galaxy $\sim 40 \operatorname{arcsec} \mathrm{W}$, no optical $z$; four other galaxies within 3-4 arcmin, $z>0.06$.

109132 - detected blue, face-on companion $\sim 1.5$ arcmin SE, SDSS J095905.12+102140.0 $\left(z=0.017938,5378 \mathrm{~km} \mathrm{~s}^{-1}\right)$.

110042 - several galaxies within 3 arcmin, all in the background or without optical redshift.

110047 - two galaxies within $\sim 1$ arcmin E without optical $z$, probably in background.

110060 - large, early-type companion $\sim 3$ arcmin S, SDSS $\mathrm{J} 102058.56+253109.8\left(z=0.020785,6231 \mathrm{~km} \mathrm{~s}^{-1}\right)$, also not detected.

111024 - large, early-type companion $\sim 1.5$ arcmin E, SDSS $\mathrm{J} 114428.32+163329.1\left(z=0.011058,3315 \mathrm{~km} \mathrm{~s}^{-1}\right)$, also not detected.

111049 - detected blue companions $\sim 1.5 \operatorname{arcmin}$ SW, SDSS $\mathrm{J} 115752.02+250254.1\left(z=0.014196,4256 \mathrm{~km} \mathrm{~s}^{-1}\right)$ and $\sim 3$ arcmin E, SDSS J115809.49+250520.0 $\left(z=0.014286,4283 \mathrm{~km} \mathrm{~s}^{-1}\right)$; also notice large spiral $\sim 4 \operatorname{arcmin}$ NE, NGC $4005\left(4464 \mathrm{~km} \mathrm{~s}^{-1}\right)$.

111066 - hint of $\mathrm{H}_{\text {I }}$ galaxy signal; blue face-on disc $\sim 2.5 \mathrm{arcmin}$ SW has $z=0.085$.

112002 - galaxy $\sim 3$ arcmin NE, SDSS J125806.06+272508.1 $\left(z=0.019237,5767 \mathrm{~km} \mathrm{~s}^{-1}\right)$ also not detected; galaxies $\sim 2.5 \operatorname{arcmin} \mathrm{NE}$ and $\sim 4 \operatorname{arcmin} \mathrm{NW}$ are in the background.

112003 - spiral galaxy $\sim 2$ arcmin $E$ has $z=0.025$; small galaxies $\sim 3$ arcmin $\mathrm{S}$ also in background.

112004 - large disc $\sim 1$ arcmin SW, SDSS J125746.16+274525.3 $\left(z=0.02051,6149 \mathrm{~km} \mathrm{~s}^{-1}\right)$ and small galaxy $\sim 2 \operatorname{arcmin}$ SE, SDSS $\mathrm{J} 125752.31+274422.7\left(z=0.023039,6907 \mathrm{~km} \mathrm{~s}^{-1}\right)$ also not detected; four other galaxies within $3 \operatorname{arcmin}, z>0.07$.

112011 - several galaxies within 3 arcmin, all in the background or without optical $z$.

112012 - galaxy $\sim 2.5 \operatorname{arcmin} \mathrm{N}$ has $z=0.023$

112016 - perhaps hint of Hi galaxy signal (offset from SDSS redshift); small, blue companion $\sim 1.5$ arcmin SW (SDSS $\mathrm{J} 125317.61+262021.4, z=0.02311,6928 \mathrm{~km} \mathrm{~s}^{-1}$ ).

$\mathbf{1 1 2 0 1 7}$ - crowded field.

112060 - two nearby galaxies in background $(\sim 2 \operatorname{arcmin} \mathrm{W}$, $z=0.025$ and $\sim 3 \operatorname{arcmin} \mathrm{N}, z=0.026$ ).

112065 - perhaps hint of $\mathrm{HI}$ signal (from galaxy and/or blue companion $\sim 3.5$ arcmin SW, SDSS J120055.36+152221.8, $z=0.017482,5241 \mathrm{~km} \mathrm{~s}^{-1}$ ).

112084 - detected face-on, blue companion $\sim 1.5 \mathrm{arcmin}$ NW, SDSS J125905.29+273839.9 $(z=0.018117,5431 \mathrm{~km}$ $\mathrm{s}^{-1}$ ); also notice smaller blue companion $\sim 2$ arcmin SE (SDSS $\mathrm{J} 125918.54+273536.9, z=0.017777,5329 \mathrm{~km} \mathrm{~s}^{-1}$ ) and galaxy $\sim 3 \operatorname{arcmin} \mathrm{W}$ (SDSS J125858.10+273540.9, $z=0.020057$, $6013 \mathrm{~km} \mathrm{~s}^{-1}$ ), both not detected.

112089 - detected blue companion $\sim 1.5 \operatorname{arcmin}$ NW, SDSS $\mathrm{J} 125020.21+264459.4\left(z=0.02373,7114 \mathrm{~km} \mathrm{~s}^{-1}\right)$; perhaps hint of $\mathrm{H}$ I signal from disc galaxy $\sim 1.5 \operatorname{arcmin} \mathrm{S}$, SDSS $\mathrm{J} 125026.59+264232.3\left(z=0.018702,5607 \mathrm{~km} \mathrm{~s}^{-1}\right)$.

112106 - detected large blue companion $\sim 1$ arcmin NE (UGC $7035, z=0.00409$ ) in board $4, \sim 1414.5 \mathrm{MHz}$.

113001 - galaxy $\sim 1.5$ arcmin NE, SDSS J130416.46+273022.9, has slightly higher redshift $\left(z=0.024, \sim 7200 \mathrm{~km} \mathrm{~s}^{-1}\right)$.

113004 - large face-on spiral $\sim 3$ arcmin SE (SDSS $\mathrm{J} 130126.12+275309.5, \quad z=0.018271, \quad 5478 \mathrm{~km} \mathrm{~s}^{-1}$ ) and small galaxy $\sim 2$ arcmin SW (SDSS J130104.82+275330.3, $z=0.019293,5784 \mathrm{~km} \mathrm{~s}^{-1}$ ) also not detected.

113012 - over 10 galaxies within 3 arcmin at slightly higher redshift $(z>0.018)$. 
113025 - four small galaxies within 1 arcmin, all without optical redshifts and most likely in the background.

113032 - early-type companion $\sim 3$ arcmin SE, SDSS $\mathrm{J} 130019.10+273313.3\left(z=0.019644,5889 \mathrm{~km} \mathrm{~s}^{-1}\right)$ also not detected; two galaxies $\sim 3$ arcmin $\mathrm{E}$ and $\sim 2$ arcmin $\mathrm{W}$ in background.

113040 - two galaxies within $\sim 1$ arcmin $\mathrm{E}, z>0.025$; blue smudge $\sim 20 \operatorname{arcsec} S$, no $z$.

$113047 \quad-$ companion $\sim 3$ arcmin NW, SDSS $\mathrm{J} 130010.41+273542.0 \quad\left(z=0.018676,5600 \mathrm{~km} \mathrm{~s}^{-1}\right) ;$ three galaxies between 1 and $3 \operatorname{arcmin} \mathrm{SE}, z=0.024-0.026$.

113051 - four galaxies within $\sim 3$ arcmin, $z=0.022-0.028$.

113060 - crowded field.

113078 - early-type $\sim 1$ arcmin SW, SDSS J130231.87 $+275607.9\left(z=0.022091,6623 \mathrm{~km} \mathrm{~s}^{-1}\right)$ also not detected.

113100 - hint of H I galaxy signal; AA2. Three small galaxies 2-4 arcmin away, $z=0.02-0.025$.

113128 - crowded field.

114008 - marginally detected companion $\sim 1.7$ arcmin W, GASS 114005 ( $z=0.015153,4543 \mathrm{~km} \mathrm{~s}^{-1}$; blend, see notes above); also notice SDSS J142807.22+255207.5 $\left(z=0.014591,4374 \mathrm{~km} \mathrm{~s}^{-1}\right)$ and NGC $5629\left(c z=4498 \mathrm{~km} \mathrm{~s}^{-1}\right.$ from NED).
114025 - detected blue companion $\sim 2.5$ arcmin E, SDSS $\mathrm{J} 142532.21+254300.1\left(z=0.013609,4080 \mathrm{~km} \mathrm{~s}^{-1}\right)$; the disc galaxy $\sim 1 \operatorname{arcmin} \mathrm{S}$ of the blue companion has $z=0.075$.

114036 - blue companion 2.5 arcmin NE, SDSS J142758.85+255158.7 $\left(z=0.015341,4599 \mathrm{~km} \mathrm{~s}^{-1}\right)$, also not detected; notice two early-type galaxies $\sim 2 \operatorname{arcmin} \mathrm{N}$ $(z=0.014)$ and $\sim 3 \operatorname{arcmin} \mathrm{E}(z=0.015)$.

114037 - detected companion, probably small blue galaxy $\sim 1.5$ arcmin NW (SDSS J142829.10+272113.4, no $z$ ); NGC 5635 $\sim 4 \operatorname{arcmin} \mathrm{N}\left(c z=4316 \mathrm{~km} \mathrm{~s}^{-1}\right.$ from NED).

114038 - small galaxy 2 arcmin E, SDSS J142723.43+255240.2 $\left(z=0.013418,4023 \mathrm{~km} \mathrm{~s}^{-1}\right)$.

114065 - early-type companion $\sim 2 \operatorname{arcmin}$ W, SDSS $\mathrm{J} 142714.65+255319.1\left(z=0.0159,4767 \mathrm{~km} \mathrm{~s}^{-1}\right)$.

114096 - a few galaxies between 2 and 3 arcmin in background, in particular SDSS J142544.50+254636.0, a blue galaxy 2 arcmin $\mathrm{W}(z=0.075)$ and SDSS J142600.40+254320.3, a large early-type $\sim 3 \operatorname{arcmin} \mathrm{S}(z=0.076$ from NED).

123011 - three galaxies between 2 and $3 \operatorname{arcmin}, z>0.04$.

This paper has been typeset from a $\mathrm{T}_{\mathrm{E}} \mathrm{X} / \mathrm{L} \mathrm{T} \mathrm{E} \mathrm{X}$ file prepared by the author. 